\title{
Parametric continuous-time linear fractional programming problems
}

Hsien-Chung Wu*

\section{"Correspondence:}

hcwu@nknucc.nknu.edu.tw

Department of Mathematics,

National Kaohsiung Normal

University, Kaohsiung, 802, Taiwan

\begin{abstract}
The main purpose of this paper is to establish the strong duality theorem for the parametric formulation of continuous-time linear fractional programming problems. We also consider the so-called extended form of continuous-time linear fractional programming problems that assume the vector-valued functions to be measurable and bounded on the time interval $[0, T]$. The by-product of the main result is the establishment of the strong duality theorem for the extended form of continuous-time linear fractional programming problems.
\end{abstract}

MSC: 46N10; $90 \mathrm{C} 26$

Keywords: parametric continuous-time linear fractional programming problems; strong duality; weak duality; weak convergence

\section{Introduction}

The theory of continuous-time linear programming problem has received considerable attention for a long time. Tyndall $[1,2]$ treated rigorously a continuous-time linear programming problem with the constant matrices, originating from the 'bottleneck problem' proposed by Bellman [3]. Levinson [4] generalized the results of Tyndall by considering the time-dependent matrices in which the functions shown in the objective and constraints were assumed to be continuous on the time interval $[0, T]$. Meidan and Perold [5], Papageorgiou [6] and Schechter [7] have also obtained some interesting results of continuoustime linear programming problem. Anderson et al. [8-10], Fleischer and Sethuraman [11] and Pullan [12-16] investigated a subclass of continuous-time linear programming problem, which is called the separated continuous-time linear programming problem and can be used to model the job-shop scheduling problems. Weiss [17] proposed a simplex-like algorithm to solve the separated continuous-time linear programming problem.

The continuous-time fractional programming problem was investigated by Zalmai [1821]. In this paper, we propose the parametric formulation of a class of continuous-time linear fractional programming problems, and we establish the weak and strong duality theorems. We first derive many useful convergent properties for the sequences of the optimal solutions of parametric continuous-time linear fractional programming problems. Using these convergent properties, we can prove the strong duality theorem for the parametric formulation of continuous-time linear fractional programming problems.

Wen and Wu [22] developed a discrete approximation method to numerically solve the continuous-time linear fractional programming problems. The weak and strong duality

(c) $2015 \mathrm{Wu}$. This article is distributed under the terms of the Creative Commons Attribution 4.0 International License (http://creativecommons.org/licenses/by/4.0/), which permits unrestricted use, distribution, and reproduction in any medium, provided you give appropriate credit to the original author(s) and the source, provide a link to the Creative Commons license, and indicate if changes were made. 
theorems were also established in Wen and Wu [22] by assuming the vector-valued functions to be continuous on the time interval $[0, T]$. If the vector-valued functions are assumed to be measurable and bounded on the time interval $[0, T]$, then the problem is called the extended form of continuous-time linear fractional programming problems. Using the strong duality theorem for the parametric formulation of continuous-time linear fractional programming problems, we can also establish the weak and strong duality theorems for the extended form of continuous-time linear fractional programming problems. The main purpose of this paper is to establish the strong duality theorem for the parametric formulation of continuous-time linear fractional programming problems. The by-product of the main result is to extend the strong duality theorem in Wen and $\mathrm{Wu}$ [22].

This paper is organized as follows. In Section 2, we introduce the primal and dual pair of continuous-time linear fractional programming problems, where the vector-valued functions are assumed to be measurable and bounded on the time interval $[0, T]$. Some elementary properties are obtained in order to prove the strong duality theorem. In Sections 3 and 4, we propose the parametric formulations of primal and dual problems, and derive some useful convergent properties for the sequences of optimal solutions of the corresponding parametric problems. In Section 5, the strong duality theorem for the parametric formulation is established. In Section 6, using the strong duality theorem for the parametric formulation, we shall establish the strong duality theorem for the extended form of continuous-time linear fractional programming problems.

\section{Continuous-time linear fractional programming problems}

For $f \in L^{2}([0, T], \mathbb{R})$, we recall

$$
\|f\|_{2}=\left(\int_{0}^{T} f^{2}(t) d t\right)^{1 / 2}
$$

Let $\mathrm{MB}([0, T], \mathbb{R})$ be the space of all measurable and bounded functions from a time interval $[0, T]$ into the Euclidean space $\mathbb{R}$. If $f \in \mathrm{MB}([0, T], \mathbb{R})$, then there exists a positive constant $C$ such that $|f(t)| \leq C$ for all $t \in[0, T]$. For $\mathbf{x}=\left(x_{1}, \ldots, x_{q}\right) \in L^{2}\left([0, T], \mathbb{R}_{+}^{q}\right)$, we mean $x_{j} \in L^{2}\left([0, T], \mathbb{R}_{+}\right)$for each $j=1, \ldots, q$, where $x_{j}$ denotes the $j$ th component of $\mathbf{x}$. Similarly, for $\mathbf{f}=\left(f_{1}, \ldots, f_{q}\right) \in \operatorname{MB}\left([0, T], \mathbb{R}^{q}\right)$, we mean $f_{j} \in \operatorname{MB}([0, T], \mathbb{R})$ for each $j=1, \ldots, q$.

The continuous-time linear fractional programming problem (CLFP) is formulated as follows:

$$
\begin{gathered}
(\text { CLFP }) \quad \max \quad \frac{f_{0}+\int_{0}^{T}(\mathbf{f}(t))^{\top} \mathbf{x}(t) d t}{h_{0}+\int_{0}^{T}(\mathbf{h}(t))^{\top} \mathbf{x}(t) d t} \\
\text { subject to } \quad B \mathbf{x}(t) \leq \mathbf{g}(t)+\int_{0}^{t} K \mathbf{x}(s) d s \quad \text { for all } t \in[0, T], \\
\quad \mathbf{x} \in L^{2}\left([0, T], \mathbb{R}_{+}^{q}\right),
\end{gathered}
$$

where $h_{0}>0, f_{0} \geq 0, \mathbf{f} \in \mathrm{MB}\left([0, T], \mathbb{R}^{q}\right), \mathbf{h} \in \mathrm{MB}\left([0, T], \mathbb{R}_{+}^{q}\right), \mathbf{g} \in \mathrm{MB}\left([0, T], \mathbb{R}_{+}^{p}\right), B=$ $\left[B_{i j}\right]_{p \times q}$ and $K=\left[K_{i j}\right]_{p \times q}$ are $p \times q$ constant matrices satisfying the following conditions:

- $K_{i j} \geq 0$ for all $i=1, \ldots, p$ and $j=1, \ldots, q$;

- $B_{i j} \geq 0$ and $\sum_{i=1}^{p} B_{i j}>0$ for all $i=1, \ldots, p$ and $j=1, \ldots, q$. 
The dual problem of primal problem (CLFP) is defined as follows:

$$
\begin{array}{ll}
\text { (DCLFP) minimize } & \beta \\
\text { subject to } \quad & B^{\top} \mathbf{z}(t)+\beta \mathbf{h}(t) \geq \mathbf{f}(t)+\int_{t}^{T} K^{\top} \mathbf{z}(s) d s \quad \text { for all } t \in[0, T], \\
& -\beta h_{0}+\int_{0}^{T}(\mathbf{g}(t))^{\top} \mathbf{z}(t) d t \leq-f_{0}, \\
& \mathbf{z} \in L^{2}\left([0, T], \mathbb{R}_{+}^{p}\right) .
\end{array}
$$

Given a primal problem (P), there are many ways to formulate the dual problem (DP) such that the weak and strong duality theorems hold true between the primal and dual pair of problems (P) and (DP). Although the dual problem (DCLFP) is not formulated as a type of continuous-time linear fractional programming problems, it does not lose the generality because the weak and strong duality theorems have been proven to hold true between the problems (CLFP) and (DCFLP) as shown in Wen and Wu [22] when the functions $\mathbf{f}$ and $\mathbf{g}$ were assumed to be continuous on $[0, T]$. In this paper, we are going to derive the weak and strong duality theorems when the functions $\mathbf{f}$ and $\mathbf{g}$ are assumed to be measurable and bounded on $[0, T]$.

Since the vector-valued function $\mathbf{g}$ is assumed to be nonnegative on $[0, T]$, it is easy to see that the primal problem (CLFP) is feasible with the trivial feasible solution $\mathbf{x}(t)=\mathbf{0}$ for all $t \in[0, T]$. Since each $f_{i}$ is bounded on $[0, T]$ for $i=1, \ldots, q$, we see that

$$
\max _{t \in[0, T]} f_{i}(t)<\infty
$$

for each $i=1, \ldots, q$. The arguments presented in Wen and Wu [22], Proposition 2.1, are still valid and guarantee the feasibility of dual problem (DCLFP) when the vector-valued functions $\mathbf{f}$ and $\mathbf{g}$ are assumed to be measurable and bounded on $[0, T]$.

Theorem 2.1 (Weak duality theorem) Considering the primal and dual pair of problems (CLFP) and (DCLFP), for any feasible solutions $\mathbf{x}$ and (z, $\beta)$ of problems (CLFP) and (DCFLP), respectively, we have

$$
\frac{f_{0}+\int_{0}^{T}(\mathbf{f}(t))^{\top} \mathbf{x}(t) d t}{h_{0}+\int_{0}^{T}(\mathbf{h}(t))^{\top} \mathbf{x}(t) d t} \leq \beta .
$$

In other words, the optimal objective value of primal problem (CLFP) is less than or equal to the optimal objective value of dual problem (DCLFP).

Proof The arguments presented in Wen and Wu [22], Theorem 2.1, are still valid when the vector-valued functions $\mathbf{f}$ and $\mathbf{g}$ are assumed to be measurable and bounded on $[0, T]$ here.

According to the approach proposed by Charnes and Cooper [23], we can transform the original problem (CLFP) into a continuous-time linear programming problem. Since $h_{0}>0$, we can define

$$
\alpha=\frac{1}{h_{0}+\int_{0}^{T}(\mathbf{h}(t))^{\top} \mathbf{x}(t) d t} \quad \text { and } \quad \mathbf{y}=\alpha \mathbf{x} .
$$


Then the original problem (CLFP) can be converted to a continuous-time linear programming problem as follows:

$$
\begin{aligned}
& \max \quad \alpha f_{0}+\int_{0}^{T}(\mathbf{f}(t))^{\top} \mathbf{y}(t) d t \\
& \text { subject to } \quad B \mathbf{y}(t) \leq \alpha \mathbf{g}(t)+\int_{0}^{t} K \mathbf{y}(s) d s \quad \text { for all } t \in[0, T] \\
& \\
& \alpha h_{0}+\int_{0}^{T}(\mathbf{h}(t))^{\top} \mathbf{y}(t) d t=1, \\
& \alpha \geq 0 \\
& \mathbf{y} \in L^{2}\left([0, T], \mathbb{R}_{+}^{q}\right) .
\end{aligned}
$$

The following result will be useful for further discussions.

Proposition 2.1 Considering the problems (AP) and (DCLFP), for any feasible solutions $(\mathbf{y}, \alpha)$ with $\alpha>0$ and $(\mathbf{z}, \beta)$ of problems (CLFP) and (DCFLP), respectively, we have

$$
\alpha f_{0}+\int_{0}^{T}(\mathbf{f}(t))^{\top} \mathbf{y}(t) d t \leq \beta .
$$

In other words, the optimal objective value of problem (AP) is less than or equal to the optimal objective value of problem (DCLFP).

Proof Let $\mathbf{x}=\mathbf{y} / \alpha$. Then $\mathbf{x}$ is a feasible solution of problem (CLFP). Using the weak duality Theorem 2.1, we have

$$
\begin{aligned}
\alpha f_{0}+\int_{0}^{T}(\mathbf{f}(t))^{\top} \mathbf{y}(t) d t & =\alpha \cdot\left(f_{0}+\int_{0}^{T}(\mathbf{f}(t))^{\top} \mathbf{x}(t) d t\right) \\
& =\frac{f_{0}+\int_{0}^{T}(\mathbf{f}(t))^{\top} \mathbf{x}(t) d t}{h_{0}+\int_{0}^{T}(\mathbf{h}(t))^{\top} \mathbf{x}(t) d t} \leq \beta
\end{aligned}
$$

This completes the proof.

We say that the vector-valued function $\mathbf{h}$ is Lipschitz continuous on $[0, T]$ if and only if there exists a constant $\gamma$ such that

$$
\left|h_{j}\left(t_{1}\right)-h_{j}\left(t_{2}\right)\right| \leq \gamma \cdot\left|t_{1}-t_{2}\right|
$$

for all $t_{1}, t_{2} \in[0, T]$ and for each $j=1, \ldots, q$.

Theorem 2.2 (Wen and Wu [22]; Strong duality theorem) Suppose that the vector-valued functions $\mathbf{f}$ and $\mathbf{g}$ are continuous on [0,T], and that the vector-valued function $\mathbf{h}$ is Lipschitz continuous on $[0, T]$. Let

$$
\begin{aligned}
& \sigma=\min \left\{B_{i j}: B_{i j}>0\right\}, \\
& \nu=\max _{j=1, \ldots, q}\left\{\sum_{i=1}^{p} K_{i j}\right\},
\end{aligned}
$$




$$
\begin{aligned}
\tau & =\max \left\{\left|f_{j}(t)\right|: j=1, \ldots, q \text { and } t \in[0, T]\right\}, \\
\zeta & =\max \left\{g_{i}(t): i=1, \ldots, p \text { and } t \in[0, T]\right\}
\end{aligned}
$$

Then there exist optimal solutions $\mathbf{x}^{*},\left(\mathbf{y}^{*}, \alpha^{*}\right)$, and $\left(\mathbf{z}^{*}, \beta^{*}\right)$ of problems (CLFP), (AP), and (DCLFP), respectively, such that $\alpha^{*}>0$ and

$$
\frac{f_{0}+\int_{0}^{T}(\mathbf{f}(t))^{\top} \mathbf{x}^{*}(t) d t}{h_{0}+\int_{0}^{T}(\mathbf{h}(t))^{\top} \mathbf{x}^{*}(t) d t}=\beta^{*}=\alpha^{*} f_{0}+\int_{0}^{T}(\mathbf{f}(t))^{\top} \mathbf{y}^{*}(t) d t,
$$

where $\mathbf{x}^{*}=\mathbf{y}^{*} / \alpha^{*}$; that is, there is no duality gap between the primal and dual pair of problems (CLFP) and (DCLFP), and the optimal objective values of problems (AP) and (DCLFP) are equal. Moreover, for $j=1, \ldots, q$ and $t \in[0, T]$, we have

$$
\alpha^{*} \leq 1 / h_{0} \quad \text { and } \quad\left|y_{j}^{*}(t)\right| \leq \frac{\zeta}{h_{0} \sigma} \cdot \exp \left(\frac{q v T}{\sigma}\right) \text { a.e. in }[0, T] .
$$

For $i=1, \ldots, p$ and $t \in[0, T]$, we have

$$
\begin{aligned}
& 0 \leq \beta^{*} \leq \frac{\zeta \cdot p \cdot \tau \cdot T \cdot \exp \left(\frac{\nu T}{\sigma}\right)+\sigma \cdot f_{0}}{\sigma \cdot h_{0}} \text { and } \\
& \left|z_{i}^{*}(t)\right| \leq \frac{\tau}{\sigma} \cdot \exp \left(\frac{\nu T}{\sigma}\right) \quad \text { a.e. in }[0, T] .
\end{aligned}
$$

We also remark that, in Wen and Wu [22], the decision variables $\mathbf{x}$ and $\mathbf{z}$ were assumed to be in $L^{\infty}\left([0, T], \mathbb{R}_{+}^{q}\right)$ and $L^{\infty}\left([0, T], \mathbb{R}_{+}^{p}\right)$, respectively. Since the proof of strong duality theorem in Wen and Wu [22] was based on the weak convergence in $L^{2}([0, T], \mathbb{R})$, it means that the strong duality theorem should be true in the situation that the decision variables $\mathbf{x}$ and $\mathbf{z}$ are assumed to be in $L^{2}\left([0, T], \mathbb{R}_{+}^{q}\right)$ and $L^{2}\left([0, T], \mathbb{R}_{+}^{p}\right)$, respectively. This is the reason why we consider the space $L^{2}([0, T], \mathbb{R})$ in this paper. The purpose of this paper is to derive the strong duality theorem when the vector-valued functions $\mathbf{f}$ and $\mathbf{g}$ are assumed to be measurable and bounded on $[0, T]$ based on the parametric formulation that will be discussed in the next section.

\section{Parametric formulation of primal problems}

Suppose that the vector-valued functions $\mathbf{f}$ and $\mathbf{g}$ in the primal problem (CLFP) can be parameterized as the vector-valued functions $\mathbf{f}_{\epsilon}$ and $\mathbf{g}_{\epsilon}$ for $\epsilon>0$ in which $\mathbf{f}_{\epsilon}$ and $\mathbf{g}_{\epsilon}$ are assumed to be continuous functions on $[0, T]$ and $\mathbf{g}_{\epsilon}(t) \geq \mathbf{0}$ for all $t \in[0, T]$. The vectorvalued function $\mathbf{h}$ is assumed to be Lipschitz continuous on $[0, T]$.

We also assume that the vector-valued functions $\mathbf{f}_{0}$ and $\mathbf{g}_{0}$ are measurable and bounded on $[0, T]$ satisfying $\mathbf{g}_{0}(t) \geq \mathbf{0}$ for all $t \in[0, T]$ and the following conditions:

$$
\begin{aligned}
& V_{\epsilon}=\left\{t \in[0, T]: \mathbf{f}_{0}(t) \neq \mathbf{f}_{\epsilon}(t)\right\} \quad \text { and } \quad \mu\left(V_{\epsilon}\right)<q \cdot \epsilon, \\
& U_{\epsilon}=\left\{t \in[0, T]: \mathbf{g}_{0}(t) \neq \mathbf{g}_{\epsilon}(t)\right\} \text { and } \mu\left(U_{\epsilon}\right)<p \cdot \epsilon,
\end{aligned}
$$

where $\mu$ denotes the Lebesgue measure. In this case, we see that

$$
\lim _{\epsilon \rightarrow 0+} \mathbf{f}_{\epsilon}(t)=\mathbf{f}_{0}(t) \quad \text { a.e. in }[0, T]
$$


and

$$
\lim _{\epsilon \rightarrow 0+} \mathbf{g}_{\epsilon}(t)=\mathbf{g}_{0}(t) \quad \text { a.e. in }[0, T] .
$$

Since the vector-valued functions $\mathbf{f}_{0}$ and $\mathbf{g}_{0}$ are measurable and bounded on $[0, T]$, there exist positive constants $\tau_{0}$ and $\zeta_{0}$ such that

$$
\left|f_{j, 0}(t)\right| \leq \tau_{0} \quad \text { for all } t \in[0, T] \text { and for all } j=1, \ldots, q
$$

and

$$
g_{i, 0}(t) \leq \zeta_{0} \quad \text { for all } t \in[0, T] \text { and for all } i=1, \ldots, p \text {, }
$$

where $f_{j, 0}$ is the $j$ th component of $\mathbf{f}_{0}$ and $g_{i, 0}$ is the $i$ th component of $\mathbf{g}_{0}$.

For each $\epsilon>0$, since the functions $\mathbf{f}_{\epsilon}$ and $\mathbf{g}_{\epsilon}$ are continuous on $[0, T]$, we define

$$
\tau_{j, \epsilon}=\max _{t \in[0, T]}\left|f_{j, \epsilon}(t)\right| \quad \text { and } \quad \tau_{\epsilon}=\max \left\{\tau_{1, \epsilon}, \ldots, \tau_{q, \epsilon}\right\}
$$

and

$$
\zeta_{i, \epsilon}=\max _{t \in[0, T]} g_{i, \epsilon}(t) \quad \text { and } \quad \zeta_{\epsilon}=\max \left\{\zeta_{1, \epsilon}, \ldots, \zeta_{p, \epsilon}\right\}
$$

where $f_{j, \epsilon}$ is the $j$ th component of $\mathbf{f}_{\epsilon}$ and $g_{i, \epsilon}$ is the $i$ th component of $\mathbf{g}_{\epsilon}$.

For $\epsilon \geq 0$, we consider the following parametric optimization problem:

$$
\begin{gathered}
\left(\mathrm{CLFP}_{\epsilon}\right) \quad \max \frac{f_{0}+\int_{0}^{T}\left(\mathbf{f}_{\epsilon}(t)\right)^{\top} \mathbf{x}(t) d t}{h_{0}+\int_{0}^{T}(\mathbf{h}(t))^{\top} \mathbf{x}(t) d t} \\
\text { subject to } \quad B \mathbf{x}(t) \leq \mathbf{g}_{\epsilon}(t)+\int_{0}^{t} K \mathbf{x}(s) d s \quad \text { for all } t \in[0, T], \\
\quad \mathbf{x} \in L^{2}\left([0, T], \mathbb{R}_{+}^{q}\right),
\end{gathered}
$$

where the vector-valued functions satisfy the following conditions:

- for each $\epsilon>0$, the vector-valued functions $\mathbf{f}_{\epsilon}$ and $\mathbf{g}_{\epsilon}$ are assumed to be continuous functions on $[0, T]$ and $\mathbf{g}_{\epsilon}(t) \geq \mathbf{0}$ for all $t \in[0, T]$;

- the vector-valued function $\mathbf{h}$ is assumed to be Lipschitz continuous on $[0, T]$;

- the vector-valued functions $\mathbf{f}_{0}$ and $\mathbf{g}_{0}$ are assumed to be measurable and bounded on $[0, T]$ satisfying $\mathbf{g}_{0}(t) \geq \mathbf{0}$ for all $t \in[0, T]$;

- for each $\epsilon>0$, we assume that $\mu\left(U_{\epsilon}\right)<p \cdot \epsilon$ and $\mu\left(V_{\epsilon}\right)<q \cdot \epsilon$ in (6) and (5), respectively.

According to the approach proposed by Charnes and Cooper [23], we can similarly transform the problem $\left(\mathrm{CLFP}_{\epsilon}\right)$ into the following parametric optimization problem:

$$
\begin{aligned}
\left(\mathrm{AP}_{\epsilon}\right) \quad \max \quad \alpha f_{0}+\int_{0}^{T}\left(\mathbf{f}_{\epsilon}(t)\right)^{\top} \mathbf{y}(t) d t & \\
& \text { subject to } B \mathbf{y}(t) \leq \alpha \mathbf{g}_{\epsilon}(t)+\int_{0}^{t} K \mathbf{y}(s) d s \quad \text { for all } t \in[0, T],
\end{aligned}
$$




$$
\begin{aligned}
& \alpha h_{0}+\int_{0}^{T}(\mathbf{h}(t))^{\top} \mathbf{y}(t) d t=1, \\
& \alpha \geq 0, \\
& \mathbf{y} \in L^{2}\left([0, T], \mathbb{R}_{+}^{q}\right) .
\end{aligned}
$$

Using Theorem 2.2, the problem $\left(\mathrm{AP}_{\epsilon}\right)$ has an optimal solution $\left(\mathbf{y}_{\epsilon}^{*}, \alpha_{\epsilon}^{*}\right)$ with $\alpha_{\epsilon}^{*}>0$ for all $\epsilon>0$.

Let $L^{\infty}([0, T], \mathbb{R})$ be the space of all measurable and essentially bounded functions from a time interval $[0, T]$ into the Euclidean space $\mathbb{R}$. For $f \in L^{\infty}([0, T], \mathbb{R})$, we recall

$$
\|f\|_{\infty}=\operatorname{ess~sup}_{t \in[0, T]}|f(t)|=\inf \{k:|f(t)| \leq k \text { a.e. }\}
$$

where the Lebesgue measure is considered. Therefore we have $|f(t)| \leq\|f\|_{\infty}$ a.e. in $[0, T]$.

Let $\left\{f_{k}\right\}_{k=1}^{\infty}$ be a sequence of functions in $L^{\infty}([0, T], \mathbb{R})$. We say that the sequence $\left\{f_{k}\right\}_{k=1}^{\infty}$ is uniformly essentially bounded on $[0, T]$ if and only if there exists a positive constant $C$ such that $\left\|f_{k}\right\|_{\infty} \leq C$ for each $k$. If $\left\{\mathbf{f}_{k}\right\}_{k=1}^{\infty}$ is a sequence of vector-valued functions, then we say that the sequence $\left\{\mathbf{f}_{k}\right\}_{k=1}^{\infty}$ is uniformly essentially bounded if and only if there exists a positive constant $C$ such that $\left\|f_{i k}\right\|_{\infty} \leq C$ for each $i$ and $k$, where $f_{i k}$ is the $i$ th component of $\mathbf{f}_{k}$.

Let $\left\{f_{k}\right\}_{k=1}^{\infty}$ be a sequence of functions in $L^{2}([0, T], \mathbb{R})$. We say that the sequence $\left\{f_{k}\right\}_{k=1}^{\infty}$ is uniformly bounded on $[0, T]$ with respect to $\|\cdot\|_{2}$ if and only if there exists a positive constant $C$ such that $\left\|f_{k}\right\|_{2} \leq C$ for each $k$. If $\left\{\mathbf{f}_{k}\right\}_{k=1}^{\infty}$ is a sequence of vector-valued functions, then we say that the sequence $\left\{\mathbf{f}_{k}\right\}_{k=1}^{\infty}$ is uniformly bounded with respect to $\|\cdot\|_{2}$ if and only if there exists a positive constant $C$ such that $\left\|f_{i k}\right\|_{2} \leq C$ for each $i$ and $k$, where $f_{i k}$ is the $i$ th component of $\mathbf{f}_{k}$.

Since each $\mathbf{f}_{\epsilon}$ is continuous on $[0, T]$, it is clearly that $\mathbf{f}_{\epsilon}$ is bounded on $[0, T]$. However, the sequence $\left\{\mathbf{f}_{\epsilon_{k}}\right\}_{k=1}^{\infty}$ is not necessarily uniformly bounded on $[0, T]$, since we may not have a constant such that each $\mathbf{f}_{\epsilon_{k}}$ is bounded by this same constant. In this paper, we shall assume that the sequence $\left\{\mathbf{f}_{\epsilon_{k}}\right\}_{k=1}^{\infty}$ is uniformly essentially bounded on $[0, T]$. It is clear that if the sequence $\left\{\mathbf{f}_{k}\right\}_{k=1}^{\infty}$ is uniformly essentially bounded on $[0, T]$, then it is also uniformly bounded on $[0, T]$ with respect to $\|\cdot\|_{2}$, since $L^{\infty}([0, T], \mathbb{R}) \subset L^{2}([0, T], \mathbb{R})$. The following lemmas are very useful.

Lemma 3.1 (Riesz and Sz.-Nagy [24], p.64) Let $\left\{f_{k}\right\}_{k=1}^{\infty}$ be a sequence in $L^{2}([0, T], \mathbb{R})$. If the sequence $\left\{f_{k}\right\}_{k=1}^{\infty}$ is uniformly bounded with respect to $\|\cdot\|_{2}$, then exists a subsequence $\left\{f_{k_{j}}\right\}_{j=1}^{\infty}$ that weakly converges to $f_{0} \in L^{2}([0, T], \mathbb{R})$. In other words, for any $g \in L^{2}([0, T], \mathbb{R})$, we have

$$
\lim _{j \rightarrow \infty} \int_{0}^{T} f_{k_{j}}(t) g(t) d t=\int_{0}^{T} f_{0}(t) g(t) d t
$$

Lemma 3.2 (Levinson [4]) If the sequence $\left\{f_{k}\right\}_{k=1}^{\infty}$ is uniformly bounded on $[0, T]$ with respect to $\|\cdot\|_{2}$ and weakly converges to $f_{0} \in L^{2}([0, T], \mathbb{R})$, then

$$
f_{0}(t) \leq \limsup _{k \rightarrow \infty} f_{k}(t) \quad \text { a.e. in }[0, T]
$$


and

$$
f_{0}(t) \geq \liminf _{k \rightarrow \infty} f_{k}(t) \quad \text { a.e. in }[0, T]
$$

The following result is useful for deriving the strong duality theorem.

Proposition 3.1 Given a sequence $\left\{\epsilon_{k}\right\}_{k=1}^{\infty}$ in $\mathbb{R}_{+} \backslash\{0\}$ with $\epsilon_{k} \rightarrow 0+$ as $k \rightarrow \infty$, suppose that the sequences $\left\{\mathbf{f}_{\epsilon_{k}}\right\}_{k=1}^{\infty}$ and $\left\{\mathbf{g}_{\epsilon_{k}}\right\}_{k=1}^{\infty}$ are uniformly essentially bounded on [0,T]. Given a sequence $\left\{\left(\mathbf{y}_{\epsilon_{k}}^{*}, \alpha_{\epsilon_{k}}^{*}\right)\right\}_{k=1}^{\infty}$ of optimal solutions of problems $\left(\mathrm{AP}_{\epsilon_{k}}\right)$ with $\alpha_{\epsilon_{k}}^{*}>0$, there exist a subsequence $\left\{\epsilon_{k_{i}}\right\}_{i=1}^{\infty}$ with $\epsilon_{k_{i}} \rightarrow 0+$ as $i \rightarrow \infty$ and a feasible solution $\left(\mathbf{y}_{0}^{*}, \alpha_{0}^{*}\right)$ of problem $\left(\mathrm{AP}_{0}\right)$ such that the subsequence $\left\{\mathbf{y}_{\epsilon_{i}}^{*}\right\}_{i=1}^{\infty}$ weakly converges to $\mathbf{y}_{0}^{*}$, and the following limits hold true:

$$
\begin{aligned}
& \lim _{i \rightarrow \infty} \alpha_{\epsilon_{k_{i}}}^{*}=\alpha_{0}^{*}, \\
& \lim _{i \rightarrow \infty} \int_{0}^{T}\left(\mathbf{f}_{0}(t)\right)^{\top} \mathbf{y}_{\epsilon_{k_{i}}}^{*}(t) d t=\int_{0}^{T}\left(\mathbf{f}_{0}(t)\right)^{\top} \mathbf{y}_{0}^{*}(t) d t, \\
& \lim _{i \rightarrow \infty} \int_{0}^{T}\left(\mathbf{f}_{\epsilon_{k_{i}}}(t)\right)^{\top} \mathbf{y}_{\epsilon_{k_{i}}}^{*}(t) d t=\int_{0}^{T}\left(\mathbf{f}_{0}(t)\right)^{\top} \mathbf{y}_{0}^{*}(t) d t .
\end{aligned}
$$

Proof Let $y_{j, \epsilon_{k}}^{*}$ be the $j$ th component of $\mathbf{y}_{\epsilon_{k}}^{*}$. Since the sequence $\left\{\mathbf{g}_{\epsilon_{k}}\right\}_{k=1}^{\infty}$ is uniformly essentially bounded, there exists a positive constant $\widehat{\zeta}$ satisfying $\left\|g_{i, \epsilon_{k}}\right\|_{\infty} \leq \widehat{\zeta}$ for each $i$ and $k$. According to (3), we have $\alpha_{\epsilon_{k}}^{*} \leq 1 / h_{0}$ and

$$
\left|y_{j, \epsilon_{k}}^{*}(t)\right| \leq \frac{\zeta_{\epsilon_{k}}}{h_{0} \sigma} \cdot \exp \left(\frac{q v T}{\sigma}\right) \leq \frac{\widehat{\zeta}}{h_{0} \sigma} \cdot \exp \left(\frac{q \nu T}{\sigma}\right) \quad \text { a.e. in }[0, T]
$$

for each $k$, which says that the sequence $\left\{\alpha_{\epsilon_{k}}^{*}\right\}_{k=1}^{\infty}$ is bounded, and the sequence $\left\{\mathbf{y}_{\epsilon_{k}}^{*}\right\}_{k=1}^{\infty}$ is uniformly essentially bounded on $[0, T]$, i.e., uniformly bounded with respect to $\|\cdot\|_{2}$ on $[0, T]$. Using Lemma 3.1, there exists a subsequence $\left\{y_{1, \epsilon_{k_{i}}^{(1)}}^{*}\right\}_{i=1}^{\infty}$ of $\left\{y_{1, \epsilon_{k}}^{*}\right\}_{k=1}^{\infty}$ that weakly converges to some $\hat{y}_{1,0} \in L^{2}([0, T], \mathbb{R})$. Using Lemma 3.1 again, there exists a subsequence $\left\{y_{2, \epsilon_{k_{i}}^{(2)}}^{*}\right\}_{i=1}^{\infty}$ of $\left\{y_{2, \epsilon_{k_{i}}^{(1)}}^{*}\right)_{i=1}^{\infty}$ that weakly converges to some $\hat{y}_{2,0} \in L^{2}([0, T], \mathbb{R})$. By induction, there exists a subsequence $\left\{y_{j, \epsilon_{k_{i}}^{(j)}}^{*}\right\}_{i=1}^{\infty}$ of $\left\{y_{j, \epsilon_{k_{i}}^{*}}^{*(j-1)}\right\}_{i=1}^{\infty}$ that weakly converges to some $\hat{y}_{j, 0} \in$ $L^{2}([0, T], \mathbb{R})$ for $j=1, \ldots, q$. Therefore we can construct a subsequence $\left\{\mathbf{y}_{\epsilon_{k_{i}}^{(q)}}^{*}\right\}_{i=1}^{\infty}$ that weakly converges to $\hat{\mathbf{y}}_{0} \in L^{2}\left([0, T], \mathbb{R}^{q}\right)$. On the other hand, since $\left\{\alpha_{(q)}^{*}\right)_{i=1}^{\infty}$ is a bounded sequence of real numbers, there exists a subsequence $\left\{\alpha_{\epsilon_{k_{i}}}^{*}\right\}_{i=1}^{\infty}$ of $\left\{\alpha_{\epsilon_{k_{i}}^{(q)}}^{*}\right\}_{i=1}^{\infty} \epsilon_{k_{i}}^{\infty}$ that converges to some $\alpha_{0}^{*}$. In this case, using the weak convergence, we have

$$
\lim _{i \rightarrow \infty} \int_{0}^{T}(\mathbf{a}(t))^{\top} \mathbf{y}_{\epsilon_{i}}^{*}(t) d t=\int_{0}^{T}(\mathbf{a}(t))^{\top} \hat{\mathbf{y}}_{0}(t) d t \quad \text { for any } \mathbf{a} \in L^{2}\left([0, T], \mathbb{R}^{q}\right)
$$

and

$$
\lim _{i \rightarrow \infty} \alpha_{\epsilon_{k_{i}}}^{*}=\alpha_{0}^{*} .
$$


Using the feasibility of $\left(\mathbf{y}_{\epsilon_{k_{i}}}^{*}, \alpha_{\epsilon_{k_{i}}}^{*}\right)$, we have

$$
1=\alpha_{\epsilon_{k_{i}}}^{*} h_{0}+\int_{0}^{T}(\mathbf{h}(t))^{\top} \mathbf{y}_{\epsilon_{k_{i}}}^{*}(t) d t
$$

and

$$
\mathbf{0} \leq B \mathbf{y}_{\epsilon_{k_{i}}}^{*}(t) \leq \alpha_{\epsilon_{k_{i}}}^{*} \mathbf{g}_{\epsilon_{k_{i}}}(t)+\int_{0}^{t} K \mathbf{y}_{\epsilon_{k_{i}}}^{*}(s) d s \quad \text { for all } t \in[0, T]
$$

By taking the limit superior from (17) and (18) and using the weak convergence and (8), we obtain

$$
\mathbf{0} \leq \limsup _{i \rightarrow \infty} B \mathbf{y}_{\epsilon_{k_{i}}}^{*}(t) \leq \alpha_{0}^{*} \mathbf{g}_{0}(t)+\int_{0}^{t} K \hat{\mathbf{y}}_{0}(s) d s \quad \text { a.e. in }[0, T]
$$

and

$$
\alpha_{0}^{*} h_{0}+\int_{0}^{T}(\mathbf{h}(t))^{\top} \hat{\mathbf{y}}_{0}(t) d t=1
$$

Using Lemma 3.2, we have

$$
\underset{i \rightarrow \infty}{\limsup } \mathbf{y}_{\epsilon_{k_{i}}}^{*}(t) \geq \hat{\mathbf{y}}_{0}(t) \geq \liminf _{i \rightarrow \infty} \mathbf{y}_{\epsilon_{k_{i}}}^{*}(t) \geq \mathbf{0} \quad \text { a.e. in }[0, T]
$$

Since $B \geq \mathbf{0}$, using (19), we also have

$$
B \hat{\mathbf{y}}_{0}(t) \leq \limsup _{i \rightarrow \infty} B \mathbf{y}_{\epsilon_{k_{i}}}^{*}(t) \leq \alpha_{0}^{*} \mathbf{g}_{0}(t)+\int_{0}^{t} K \hat{\mathbf{y}}_{0}(s) d s \quad \text { a.e. in }[0, T]
$$

Let $N_{0}$ be the subset of $[0, T]$ such that the inequality (22) is violated, and let $N_{1}$ be the subset of $[0, T]$ such that $\hat{\mathbf{y}}_{0}(t) \nsupseteq \mathbf{0}$. We define $N=N_{0} \cup N_{1}$. Then, from (21), we see that the set $N$ has measure zero. Now, we define

$$
\mathbf{y}_{0}^{*}(t)= \begin{cases}\hat{\mathbf{y}}_{0}(t) & \text { if } t \notin N \\ \mathbf{0} & \text { if } t \in N\end{cases}
$$

Then we see that $\mathbf{y}_{0}^{*}(t) \geq \mathbf{0}$ for all $t \in[0, T]$ and $\mathbf{y}_{0}^{*}(t)=\hat{\mathbf{y}}_{0}(t)$ a.e. in $[0, T]$.

- For $t \notin N$, from (22), we have

$$
B \mathbf{y}_{0}^{*}(t)=B \hat{\mathbf{y}}_{0}(t) \leq \alpha_{0}^{*} \mathbf{g}_{0}(t)+\int_{0}^{t} K \hat{\mathbf{y}}_{0}(s) d s=\alpha_{0}^{*} \mathbf{g}_{0}(t)+\int_{0}^{t} K \mathbf{y}_{0}^{*}(s) d s
$$

- For $t \in N$, using (19), we also have

$$
B \mathbf{y}_{0}^{*}(t)=\mathbf{0} \leq \alpha_{0}^{*} \mathbf{g}_{0}(t)+\int_{0}^{t} K \hat{\mathbf{y}}_{0}(s) d s=\alpha_{0}^{*} \mathbf{g}_{0}(t)+\int_{0}^{t} K \mathbf{y}_{0}^{*}(s) d s
$$


From (20), we can obtain

$$
\alpha_{0}^{*} h_{0}+\int_{0}^{T}(\mathbf{h}(t))^{\top} \mathbf{y}_{0}^{*}(t) d t=\alpha_{0}^{*} h_{0}+\int_{0}^{T}(\mathbf{h}(t))^{\top} \hat{\mathbf{y}}_{0}(t) d t=1
$$

This shows that $\left(\mathbf{y}_{0}^{*}, \alpha_{0}^{*}\right)$ is a feasible solution of problem $\left(\operatorname{AP}_{0}\right)$. Since $\mathbf{y}_{0}^{*}(t)=\hat{\mathbf{y}}_{0}(t)$ a.e. in $[0, T]$, from (15), we see that the subsequence $\left\{\mathbf{y}_{\epsilon_{k}}^{*}\right\}_{i=1}^{\infty}$ weakly converges to $\mathbf{y}_{0}^{*}$.

Since $\mathbf{f}_{0}$ is assumed to be measurable and bounded on $[0, T]$, it follows that $\mathbf{f}_{0} \in$ $L^{2}\left([0, T], \mathbb{R}^{q}\right)$. Since $\left\{\mathbf{y}_{\epsilon_{k_{i}}^{*}}^{*}\right\}_{i=1}^{\infty}$ weakly converges to $\mathbf{y}_{0}^{*}$, this proves the limit (13). We remain to prove the limit (14). Since the sequence $\left\{\mathbf{f}_{\epsilon_{k}}(t)\right\}$ is uniformly essentially bounded on $[0, T]$, there exists a positive constant $\widehat{\tau}$ such that $\left\|f_{j, \epsilon_{k}}\right\|_{\infty} \leq \widehat{\tau}$ for each $j$ and $k$. Now we have

$$
\begin{aligned}
& \left|\int_{0}^{T}\left(\mathbf{f}_{\epsilon_{k_{i}}}(t)\right)^{\top} \mathbf{y}_{\epsilon_{k_{i}}}^{*}(t) d t-\int_{0}^{T}\left(\mathbf{f}_{0}(t)\right)^{\top} \mathbf{y}_{\epsilon_{k_{i}}}^{*}(t) d t\right| \\
& \quad=\left|\int_{0}^{T}\left[\mathbf{f}_{\epsilon_{k_{i}}}(t)-\mathbf{f}_{0}(t)\right]^{\top} \mathbf{y}_{\epsilon_{k_{i}}}^{*}(t) d t\right| \\
& \quad=\left|\int_{{V_{\epsilon_{k}}}}\left[\mathbf{f}_{\epsilon_{k_{i}}}(t)-\mathbf{f}_{0}(t)\right]^{\top} \mathbf{y}_{\epsilon_{k_{i}}}^{*}(t) d t\right| \quad \text { (using assumption (5)) } \\
& \quad \leq \mu\left(V_{\epsilon_{k_{i}}}\right) \cdot \sum_{j=1}^{q}\left(\widehat{\tau}+\tau_{0}\right) \cdot\left\|y_{j, \epsilon_{k_{i}}}^{*}\right\|_{\infty} \quad\left(\tau_{0}\right. \text { satisfies (9)) } \\
& \quad<q \cdot \epsilon_{k_{i}} \cdot\left(\widehat{\tau}+\tau_{0}\right) \cdot \sum_{j=1}^{q}\left\|y_{j, \epsilon_{k_{i}}}^{*}\right\|_{\infty} \quad \text { (using assumption (5)) }
\end{aligned}
$$

and

$$
\begin{aligned}
& \left|\int_{0}^{T}\left(\mathbf{f}_{\epsilon_{k_{i}}}(t)\right)^{\top} \mathbf{y}_{\epsilon_{k_{i}}}^{*}(t) d t-\int_{0}^{T}\left(\mathbf{f}_{0}(t)\right)^{\top} \mathbf{y}_{0}^{*}(t) d t\right| \\
& \leq\left|\int_{0}^{T}\left(\mathbf{f}_{\epsilon_{k_{i}}}(t)\right)^{\top} \mathbf{y}_{\epsilon_{k_{i}}}^{*}(t) d t-\int_{0}^{T}\left(\mathbf{f}_{0}(t)\right)^{\top} \mathbf{y}_{\epsilon_{k_{i}}}^{*}(t) d t\right| \\
& \quad+\left|\int_{0}^{T}\left(\mathbf{f}_{0}(t)\right)^{\top} \mathbf{y}_{\epsilon_{k_{i}}}^{*}(t) d t-\int_{0}^{T}\left(\mathbf{f}_{0}(t)\right)^{\top} \mathbf{y}_{0}^{*}(t) d t\right| \\
& \quad<q \cdot \epsilon_{k_{i}} \cdot\left(\widehat{\tau}+\tau_{0}\right) \cdot \sum_{j=1}^{q}\left\|y_{j, \epsilon_{k_{i}}}^{*}\right\|_{\infty}+\left|\int_{0}^{T}\left(\mathbf{f}_{0}(t)\right)^{\top} \mathbf{y}_{\epsilon_{k_{i}}}^{*}(t) d t-\int_{0}^{T}\left(\mathbf{f}_{0}(t)\right)^{\top} \mathbf{y}_{0}^{*}(t) d t\right| .
\end{aligned}
$$

Since $\epsilon_{k_{i}} \rightarrow 0+$ as $i \rightarrow \infty$, the limit (14) follows from the limit (13) immediately, and the proof is complete.

\section{Parametric formulation of dual problems}

For any $\epsilon \geq 0$, we consider the following parametric optimization problem:

$$
\begin{array}{lll}
\left(\mathrm{DCLFP}_{\epsilon}\right) \quad \operatorname{minimize} & \beta \\
\text { subject to } & B^{\top} \mathbf{z}(t)+\beta \mathbf{h}(t) \geq \mathbf{f}_{\epsilon}(t)+\int_{t}^{T} K^{\top} \mathbf{z}(s) d s \\
& \quad \text { for all } t \in[0, T],
\end{array}
$$




$$
\begin{aligned}
& -\beta h_{0}+\int_{0}^{T}\left(\mathbf{g}_{\epsilon}(t)\right)^{\top} \mathbf{z}(t) d t \leq-f_{0}, \\
& \mathbf{z} \in L^{2}\left([0, T], \mathbb{R}_{+}^{p}\right) .
\end{aligned}
$$

We see that $\left(\operatorname{CLFP}_{\epsilon}\right)$ and $\left(\operatorname{DCLFP}_{\epsilon}\right)$ are a primal and dual pair of problems for any $\epsilon \geq 0$.

Lemma 4.1 For each $\epsilon>0$, we define the real-valued function

$$
\rho_{\epsilon}(t)=\frac{\tau_{\epsilon}}{\sigma} \cdot \exp \left[\frac{\nu(T-t)}{\sigma}\right]
$$

on $[0, T]$. If $\left(\hat{\mathbf{z}}_{\epsilon}, \bar{\beta}_{\epsilon}\right)$ is a feasible solution of the dual problem $\left(\mathrm{DCLFP}_{\epsilon}\right)$, then there exists a feasible solution $\left(\overline{\mathbf{z}}_{\epsilon}(t), \bar{\beta}_{\epsilon}\right)$ of dual problem $\left(\mathrm{DCLFP}_{\epsilon}\right)$ such that $\overline{\mathbf{z}}_{\epsilon}(t) \leq \hat{\mathbf{z}}_{\epsilon}(t)$ and $\bar{z}_{i, \epsilon}(t) \leq$ $\rho_{\epsilon}(t)$ for each $i$ and $t \in[0, T]$, where $\bar{z}_{i, \epsilon}$ is the ith component of $\overline{\mathbf{z}}_{\epsilon}$.

Proof Since $\mathbf{f}_{\epsilon}$ and $\mathbf{g}_{\epsilon}$ are assumed to be continuous on [0,T] for $\epsilon>0$, the result follows from Wen and Wu [22], Lemma 5.3, immediately.

Proposition 4.1 Given a sequence $\left\{\epsilon_{k}\right\}_{k=1}^{\infty}$ in $\mathbb{R}_{+} \backslash\{0\}$ with $\epsilon_{k} \rightarrow 0+$ as $k \rightarrow \infty$, suppose that the sequences $\left\{\mathbf{f}_{\epsilon_{k}}\right\}_{k=1}^{\infty}$ and $\left\{\mathbf{g}_{\epsilon_{k}}\right\}_{k=1}^{\infty}$ are uniformly essentially bounded on [0,T]. Given a sequence $\left\{\left(\mathbf{z}_{\epsilon_{k}}^{*}, \beta_{\epsilon_{k}}^{*}\right)\right\}_{k=1}^{\infty}$ of optimal solutions of problems $\left(\mathrm{DCLFP}_{\epsilon_{k}}\right)$, there exist a subsequence $\left\{\epsilon_{k_{j}}\right\}_{j=1}^{\infty}$ with $\epsilon_{k_{j}} \rightarrow 0+$ as $j \rightarrow \infty$ and a feasible solution $\left(\mathbf{z}_{0}^{*}, \beta_{0}^{*}\right)$ of problem $\left(\mathrm{DCLFP}_{0}\right)$ such that the following limit holds true:

$$
\lim _{j \rightarrow \infty} \beta_{\epsilon_{k_{j}}}^{*}=\beta_{0}^{*} .
$$

Proof For each $\epsilon>0$, since $\mathbf{f}_{\epsilon}$ and $\mathbf{g}_{\epsilon}$ are continuous on [0,T], Theorem 2.2 says that the dual problem $\left(\operatorname{DCLFP}_{\epsilon}\right)$ has an optimal solution $\left(\mathbf{z}_{\epsilon}^{*}, \beta_{\epsilon}^{*}\right)$, where $\mathbf{z}_{\epsilon}^{*}=\left(z_{1, \epsilon}^{*}, \ldots, z_{p, \epsilon}^{*}\right)$. Now, we consider the real-valued function $\rho_{\epsilon}$ defined in (26), and write $\rho_{\epsilon}$ as a $p$-dimensional vector-valued function with all entries $\rho_{\epsilon}$. Using Lemma 4.1, there exists a feasible solution $\left(\hat{\mathbf{z}}_{\epsilon}, \beta_{\epsilon}^{*}\right)$ of $\left(\mathrm{DCLFP}_{\epsilon}\right)$ such that

$$
\hat{\mathbf{z}}_{\epsilon}(t) \leq \mathbf{z}_{\epsilon}^{*}(t) \quad \text { and } \quad \hat{\mathbf{z}}_{\epsilon}(t) \leq \boldsymbol{\rho}_{\epsilon}(t) \quad \text { for all } t \in[0, T]
$$

Since the sequence $\left\{\mathbf{f}_{\epsilon_{k}}(t)\right\}$ is uniformly essentially bounded on $[0, T]$, there exists a positive constant $\widehat{\tau}$ such that $\left\|f_{j, \epsilon_{k}}\right\|_{\infty} \leq \widehat{\tau}$ for each $j$ and $k$. Let

$$
\tau^{*}=\max \left\{\widehat{\tau}, \tau_{0}\right\},
$$

where $\tau_{0}$ satisfies (9). In this case, we define the real-valued function

$$
\rho_{0}(t)=\frac{\tau^{*}}{\sigma} \cdot \exp \left[\frac{\nu(T-t)}{\sigma}\right]
$$

on $[0, T]$. We also write $\rho_{0}$ as a $p$-dimensional vector-valued function with all entries $\rho_{0}$. Then

$$
\hat{\mathbf{z}}_{\epsilon}(t) \leq \boldsymbol{\rho}_{\epsilon}(t) \leq \boldsymbol{\rho}_{0}(t) \quad \text { for all } t \in[0, T] \text { and for all } \epsilon>0
$$


and, from (9),

$$
\left|f_{j, 0}(t)\right| \leq \tau^{*} \quad \text { for all } t \in[0, T] \text { and for all } j=1, \ldots, n \text {. }
$$

From (28), we can obtain

$$
\sigma \rho_{0}(t)=\tau^{*}+v \cdot \int_{t}^{T} \rho_{0}(s) d s \quad \text { for all } t \in[0, T] .
$$

The assumption of $B_{i j}$ says that $\sum_{i} B_{i j} \geq \sigma$ for each $j$. By (30) and (29), for each $j=1, \ldots, n$, we have

$$
\begin{aligned}
\sum_{i} B_{i j} \rho_{0}(t) & \geq \sigma \rho_{0}(t) \geq\left|f_{j, 0}(t)\right|+\sum_{i} \int_{t}^{T}\left|K_{i j}\right| \rho_{0}(s) d s \\
& \geq f_{j, 0}(t)+\sum_{i} \int_{t}^{T} K_{i j} \rho_{0}(s) d s \quad \text { for all } t \in[0, T],
\end{aligned}
$$

which shows that

$$
B^{\top} \boldsymbol{\rho}_{0}(t) \geq \mathbf{f}_{0}(t)+\int_{t}^{T} K^{\top} \boldsymbol{\rho}_{0}(s) d s \quad \text { for all } t \in[0, T] .
$$

Since $\beta_{\epsilon_{k}}^{*} \geq 0$ and the vector-valued function $\mathbf{h}$ is nonnegative, we have

$$
B^{\top} \boldsymbol{\rho}_{0}(t)+\beta_{\epsilon_{k}}^{*} \mathbf{h}(t) \geq B^{\top} \boldsymbol{\rho}_{0}(t) \geq \mathbf{f}_{0}(t)+\int_{t}^{T} K^{\top} \boldsymbol{\rho}_{0}(s) d s \quad \text { for all } t \in[0, T] .
$$

Since the sequences $\left\{\mathbf{f}_{\epsilon_{k}}\right\}_{k=1}^{\infty}$ and $\left\{\mathbf{g}_{\epsilon_{k}}\right\}_{k=1}^{\infty}$ are uniformly essentially bounded on $[0, T]$, from (4), we see that the sequence $\left\{\beta_{\epsilon_{k}}^{*}\right\}_{k=1}^{\infty}$ is bounded, and the sequence $\left\{\mathbf{z}_{\epsilon_{k}}^{*}\right\}_{k=1}^{\infty}$ is uniformly essentially bounded on $[0, T]$. From (27), we also see that $\left\{\hat{\mathbf{z}}_{\epsilon_{k}}\right\}_{k=1}^{\infty}$ is uniformly essentially bounded on $[0, T]$, i.e., uniformly bounded with respect to $\|\cdot\|_{2}$ on $[0, T]$. Using Lemma 3.1 and the induction argument given in the proof of Proposition 3.1, there exists a subsequence $\left\{\hat{\mathbf{z}}_{\epsilon_{k_{j}}^{(p)}}\right\}_{j=1}^{\infty}$ that weakly converges to some $\hat{\mathbf{z}}_{0} \in L^{2}\left([0, T], \mathbb{R}^{p}\right)$. On the other hand, since the sequence $\left\{\beta_{\epsilon_{k_{j}}^{(p)}}^{*}\right\}_{j=1}^{\infty}$ is bounded, there exists a subsequence $\left\{\beta_{\epsilon_{k_{j}}}^{*}\right\}_{j=1}^{\infty}$ of $\left\{\beta_{\epsilon_{k_{j}}^{(p)}}^{*}\right\}_{j=1}^{\infty}$ that converges to some $\beta_{0}^{*}$. Therefore we have

$$
\lim _{j \rightarrow \infty} \int_{0}^{T} \hat{\mathbf{z}}_{\epsilon_{j}}^{\top}(t) \mathbf{c}(t) d t=\int_{0}^{T} \hat{\mathbf{z}}_{0}^{\top}(t) \mathbf{c}(t) d t \quad \text { for any } \mathbf{c} \in L^{2}\left([0, T], \mathbb{R}^{p}\right)
$$

and

$$
\lim _{j \rightarrow \infty} \beta_{\epsilon_{j}}^{*}=\beta_{0}^{*}
$$

By the feasibility of $\left(\hat{\mathbf{z}}_{\epsilon_{k_{j}}}, \beta_{\epsilon_{k_{j}}}^{*}\right)$, we have

$$
B^{\top} \hat{\mathbf{z}}_{\epsilon_{k_{j}}}(t)+\beta_{\epsilon_{k_{j}}}^{*} \mathbf{h}(t) \geq \mathbf{f}_{\epsilon_{k_{j}}}(t)+\int_{t}^{T} K^{\top} \hat{\mathbf{z}}_{\epsilon_{k_{j}}}(s) d s \quad \text { for all } t \in[0, T]
$$


and

$$
-\beta_{\epsilon_{k_{j}}}^{*} h_{0}+\int_{0}^{T} \mathbf{g}_{\epsilon_{k_{j}}}^{\top}(t) \hat{\mathbf{z}}_{\epsilon_{k_{j}}}(t) d t \leq-f_{0}
$$

Since $\mathbf{g}_{0}$ is assumed to be measurable and bounded on $[0, T]$, it follows that $\mathbf{g}_{0} \in$ $L^{2}\left([0, T], \mathbb{R}^{q}\right)$. From (33), we also have

$$
\lim _{j \rightarrow \infty} \int_{0}^{T}\left(\mathbf{g}_{0}(t)\right)^{\top} \hat{\mathbf{z}}_{\epsilon_{j}}(t) d t=\int_{0}^{T}\left(\mathbf{g}_{0}(t)\right)^{\top} \hat{\mathbf{z}}_{0}(t) d t
$$

Since the sequence $\left\{\mathbf{g}_{\epsilon_{k}}\right\}$ is uniformly essentially bounded on $[0, T]$, there exists a positive constant $\widehat{\zeta}$ such that $\left\|g_{i, \epsilon_{k}}\right\|_{\infty} \leq \widehat{\zeta}$ for each $i$ and $k$. Now we have

$$
\begin{aligned}
& \left|\int_{0}^{T}\left(\mathbf{g}_{\epsilon_{k_{j}}}(t)\right)^{\top} \hat{\mathbf{z}}_{\epsilon_{k_{j}}}(t) d t-\int_{0}^{T}\left(\mathbf{g}_{0}(t)\right)^{\top} \hat{\mathbf{z}}_{\epsilon_{k_{j}}}(t) d t\right| \\
& \quad=\left|\int_{0}^{T}\left[\mathbf{g}_{\epsilon_{k_{j}}}(t)-\mathbf{g}_{0}(t)\right]^{\top} \hat{\mathbf{z}}_{\epsilon_{k_{j}}}(t) d t\right| \\
& \quad=\left|\int_{U_{\epsilon_{k_{j}}}}\left[\mathbf{g}_{\epsilon_{k_{j}}}(t)-\mathbf{g}_{0}(t)\right]^{\top} \hat{\mathbf{z}}_{\epsilon_{k_{j}}}(t) d t\right| \quad \text { (using assumption (6)) } \\
& \quad \leq \mu\left(U_{\epsilon_{k_{j}}}\right) \cdot \sum_{i=1}^{q}\left(\widehat{\zeta}+\zeta_{0}\right) \cdot\left\|z_{i, \epsilon_{k_{j}}}\right\|_{\infty} \quad\left(\zeta_{0}\right. \text { satisfies (10)) } \\
& \quad<p \cdot \epsilon_{k_{j}} \cdot\left(\widehat{\zeta}+\zeta_{0}\right) \cdot \sum_{i=1}^{q}\left\|z_{i, \epsilon_{k_{j}}}\right\|_{\infty} \quad \text { (using assumption (6)) }
\end{aligned}
$$

and

$$
\begin{aligned}
& \left|\int_{0}^{T}\left(\mathbf{g}_{\epsilon_{k_{j}}}(t)\right)^{\top} \mathbf{z}_{\epsilon_{k_{j}}}(t) d t-\int_{0}^{T}\left(\mathbf{g}_{0}(t)\right)^{\top} \hat{\mathbf{z}}_{0}(t) d t\right| \\
& \leq\left|\int_{0}^{T}\left(\mathbf{g}_{\epsilon_{j}}(t)\right)^{\top} \hat{\mathbf{z}}_{\epsilon_{k_{j}}}(t) d t-\int_{0}^{T}\left(\mathbf{g}_{0}(t)\right)^{\top} \hat{\mathbf{z}}_{\epsilon_{k_{j}}}(t) d t\right| \\
& \quad+\left|\int_{0}^{T}\left(\mathbf{g}_{0}(t)\right)^{\top} \hat{\mathbf{z}}_{\epsilon_{k_{j}}}(t) d t-\int_{0}^{T}\left(\mathbf{g}_{0}(t)\right)^{\top} \hat{\mathbf{z}}_{0}(t) d t\right| \\
& \quad<p \cdot \epsilon_{k_{j}} \cdot\left(\widehat{\zeta}+\zeta_{0}\right) \cdot \sum_{i=1}^{q}\left\|z_{i, \epsilon_{k_{j}}}\right\|_{\infty}+\left|\int_{0}^{T}\left(\mathbf{g}_{0}(t)\right)^{\top} \hat{\mathbf{z}}_{\epsilon_{k_{j}}}(t) d t-\int_{0}^{T}\left(\mathbf{g}_{0}(t)\right)^{\top} \hat{\mathbf{z}}_{0}(t) d t\right| .
\end{aligned}
$$

Since $\epsilon_{k_{j}} \rightarrow 0+$ as $i \rightarrow \infty$, using (37), we obtain

$$
\lim _{j \rightarrow \infty} \int_{0}^{T} \mathbf{g}_{\epsilon_{k_{j}}}^{\top}(t) \hat{\mathbf{z}}_{\epsilon_{k_{j}}}(t) d t=\int_{0}^{T} \mathbf{g}_{0}^{\top}(t) \hat{\mathbf{z}}_{0}(t) d t
$$

By taking the limit inferior from (35) and (36), and using the weak convergence and the limits (7), (8), and (38), we obtain

$$
\left[\liminf _{j \rightarrow \infty} B^{\top} \hat{\mathbf{z}}_{\epsilon_{j}}(t)\right]+\beta_{0}^{*} \mathbf{h}(t) \geq \mathbf{f}_{0}(t)+\int_{t}^{T} K^{\top} \hat{\mathbf{z}}_{0}(s) d s \quad \text { a.e. in }[0, T]
$$


and

$$
-\beta_{0}^{*} h_{0}+\int_{0}^{T} \mathbf{g}_{0}^{\top}(t) \hat{\mathbf{z}}_{0}(t) d t \leq-f_{0}
$$

Using Lemma 3.2, we also have

$$
\hat{\mathbf{z}}_{0}(t) \geq \liminf _{j \rightarrow \infty} \hat{\mathbf{z}}_{\epsilon_{k_{j}}}(t) \geq \mathbf{0} \quad \text { a.e. in }[0, T]
$$

and

$$
\hat{\mathbf{z}}_{0}(t) \leq \limsup _{j \rightarrow \infty} \hat{\mathbf{z}}_{\epsilon_{j}}(t) \quad \text { a.e. in }[0, T] .
$$

Since $\hat{\mathbf{z}}_{\epsilon_{j}}(t) \leq \boldsymbol{\rho}_{0}(t)$ for all $t \in[0, T]$, from (42), we also have

$$
\hat{\mathbf{z}}_{0}(t) \leq \rho_{0}(t) \quad \text { a.e. in }[0, T]
$$

Since $B \geq \mathbf{0}$, using (39) and (41), we have

$$
\begin{aligned}
B^{\top} \hat{\mathbf{z}}_{0}(t)+\beta_{0}^{*} \mathbf{h}(t) & \geq\left[\liminf _{j \rightarrow \infty} B^{\top} \hat{\mathbf{z}}_{\epsilon_{j}}(t)\right]+\beta_{0}^{*} \mathbf{h}(t) \\
& \geq \mathbf{f}_{0}(t)+\int_{t}^{T} K^{\top} \hat{\mathbf{z}}_{0}(s) d s \quad \text { a.e. in }[0, T] .
\end{aligned}
$$

Let $N_{0}$ be the subset of $[0, T]$ such that the inequality (44) is violated, and let $N_{1}$ be the subset of $[0, T]$ such that $\hat{\mathbf{z}}_{0}(t) \nsupseteq \mathbf{0}$. We define $N=N_{0} \cup N_{1}$. Then, from (41), we see that the set $N$ has measure zero. Now we define

$$
\mathbf{z}_{0}^{*}(t)= \begin{cases}\hat{\mathbf{z}}_{0}(t) & \text { if } t \notin N, \\ \rho_{0}(t) & \text { if } t \in N .\end{cases}
$$

Then $\mathbf{z}_{0}^{*}(t) \geq \mathbf{0}$ for all $t \in[0, T]$ and $\mathbf{z}_{0}^{*}(t)=\hat{\mathbf{z}}_{0}(t)$ a.e. in $[0, T]$. We are going to claim that $\left(\mathbf{z}_{0}^{*}, \beta_{0}^{*}\right)$ is a feasible solution of dual Problem $\left(\mathrm{DCLP}_{0}\right)$. From (40), we have

$$
-\beta_{0}^{*} h_{0}+\int_{0}^{T} \mathbf{g}_{0}^{\top}(t) \mathbf{z}_{0}^{*}(t) d t=-\beta_{0}^{*} h_{0}+\int_{0}^{T} \mathbf{g}_{0}^{\top}(t) \hat{\mathbf{z}}_{0}(t) d t \leq-f_{0} .
$$

- Suppose that $t \notin N$. From (44), we have

$$
\begin{aligned}
B^{\top} \mathbf{z}_{0}^{*}(t)+\beta_{0}^{*} \mathbf{h}(t) & =B^{\top} \hat{\mathbf{z}}_{0}(t)+\beta_{0}^{*} \mathbf{h}(t) \\
& \geq \mathbf{f}_{0}(t)+\int_{t}^{T} K^{\top} \hat{\mathbf{z}}_{0}(s) d s=\mathbf{f}_{0}(t)+\int_{t}^{T} K^{\top} \mathbf{z}_{0}^{*}(s) d s .
\end{aligned}
$$

- Suppose that $t \in N$. From (43), we see that

$$
\mathbf{z}_{0}^{*}(t) \leq \rho_{0}(t) \quad \text { a.e. in }[0, T] .
$$


Using (32), (34), and (46), we also have

$$
\begin{aligned}
B^{\top} \mathbf{z}_{0}^{*}(t)+\beta_{0}^{*} \mathbf{h}(t) & =B^{\top} \boldsymbol{\rho}_{0}(t)+\beta_{0}^{*} \mathbf{h}(t) \\
& \geq \mathbf{f}_{0}(t)+\int_{t}^{T} K^{\top} \boldsymbol{\rho}_{0}(s) d s \\
& \geq \mathbf{f}_{0}(t)+\int_{t}^{T} K^{\top} \mathbf{z}_{0}^{*}(s) d s .
\end{aligned}
$$

From (45), we conclude that $\left(\mathbf{z}_{0}^{*}, \beta_{0}^{*}\right)$ is indeed a feasible solution of dual problem $\left(\mathrm{DCLP}_{0}\right)$. This completes the proof.

\section{Strong duality theorem for the parametric formulation}

According to Propositions 3.1 and 4.1, we are going to establish the strong duality theorem based on the parametric formulations of continuous-time linear fractional programming problems $\left(\mathrm{CLFP}_{\epsilon_{k}}\right)$ and $\left(\mathrm{DCLFP}_{\epsilon_{k}}\right)$ for $\epsilon_{k} \rightarrow 0+$ as $k \rightarrow \infty$.

Theorem 5.1 (Strong duality theorem) Given a sequence $\left\{\epsilon_{k}\right\}_{k=1}^{\infty}$ in $\mathbb{R}_{+} \backslash\{0\}$ with $\epsilon_{k} \rightarrow 0+$ as $k \rightarrow \infty$, suppose that the sequences of functions $\left\{\mathbf{f}_{\epsilon_{k}}\right\}$ and $\left\{\mathbf{g}_{\epsilon_{k}}\right\}$ are uniformly essentially bounded on $[0, T]$. Then the following statements hold true.

(i) For each $k$, there exist optimal solutions $\mathbf{x}_{\epsilon_{k}}^{*},\left(\mathbf{y}_{\epsilon_{k}}^{*}, \alpha_{\epsilon_{k}}^{*}\right)$, and $\left(\mathbf{z}_{\epsilon_{k}}^{*}, \beta_{\epsilon_{k}}^{*}\right)$ of problems $\left(\mathrm{CLFP}_{\epsilon_{k}}\right),\left(\mathrm{AP}_{\epsilon_{k}}\right)$, and $\left(\mathrm{DCLFP}_{\epsilon_{k}}\right)$, respectively, such that

$$
\frac{f_{0}+\int_{0}^{T} \mathbf{f}_{\epsilon_{k}}^{\top}(t) \mathbf{x}_{\epsilon_{k}}^{*}(t) d t}{h_{0}+\int_{0}^{T}(\mathbf{h}(t))^{\top} \mathbf{x}_{\epsilon_{k}}^{*}(t) d t}=\beta_{\epsilon_{k}}^{*}=\alpha_{\epsilon_{k}}^{*} f_{0}+\int_{0}^{T} \mathbf{f}_{\epsilon_{k}}^{\top}(t) \mathbf{y}_{\epsilon_{k}}^{*}(t) d t,
$$

where $\alpha_{\epsilon_{k}}^{*}>0$ and $\mathbf{x}_{\epsilon_{k}}^{*}=\mathbf{y}_{\epsilon_{k}}^{*} / \alpha_{\epsilon_{k}}^{*}$.

(ii) There exist a subsequence $\left\{\epsilon_{k_{j}}\right\}_{j=1}^{\infty}$ of $\left\{\epsilon_{k}\right\}_{k=1}^{\infty}$ with $\epsilon_{k_{j}} \rightarrow 0+$ as $j \rightarrow \infty$ and the feasible solutions $\left(\mathbf{y}_{0}^{*}, \alpha_{0}^{*}\right)$ and $\left(\mathbf{z}_{0}^{*}, \beta_{0}^{*}\right)$ of problems $\left(\mathrm{AP}_{0}\right)$ and $\left(\mathrm{DCLFP}_{0}\right)$, respectively, such that the following limits hold true:

$$
\begin{aligned}
& \lim _{j \rightarrow \infty} \alpha_{\epsilon_{k_{j}}}^{*}=\alpha_{0}^{*}, \\
& \lim _{j \rightarrow \infty} \beta_{\epsilon_{k_{j}}}^{*}=\beta_{0}^{*}, \\
& \lim _{j \rightarrow \infty} \int_{0}^{T} \mathbf{f}_{\epsilon_{k_{j}}}^{\top}(t) \mathbf{y}_{\epsilon_{k_{j}}}^{*}(t) d t=\int_{0}^{T}\left(\mathbf{f}_{0}(t)\right)^{\top} \mathbf{y}_{0}^{*}(t) d t,
\end{aligned}
$$

and the following equality is satisfied:

$$
\alpha_{0}^{*} f_{0}+\int_{0}^{T}\left(\mathbf{f}_{0}(t)\right)^{\top} \mathbf{y}_{0}^{*}(t) d t=\beta_{0}^{*}
$$

If $\alpha_{0}^{*}>0$, then $\mathbf{x}_{0}^{*}=\mathbf{y}_{0}^{*} / \alpha_{0}^{*},\left(\mathbf{y}_{0}^{*}, \alpha_{0}^{*}\right)$, and $\left(\mathbf{z}_{0}^{*}, \beta_{0}^{*}\right)$ are the optimal solutions of problems $\left(\mathrm{CLFP}_{0}\right),\left(\mathrm{AP}_{0}\right)$, and $\left(\mathrm{DCLFP}_{0}\right)$, respectively, such that the following equalities hold true:

$$
\alpha_{0}^{*} f_{0}+\int_{0}^{T}\left(\mathbf{f}_{0}(t)\right)^{\top} \mathbf{y}_{0}^{*}(t) d t=\beta_{0}^{*}=\frac{f_{0}+\int_{0}^{T}\left(\mathbf{f}_{0}(t)\right)^{\top} \mathbf{x}_{0}^{*}(t) d t}{h_{0}+\int_{0}^{T}(\mathbf{h}(t))^{\top} \mathbf{x}_{0}^{*}(t) d t}
$$


(iii) We further assume that the vector-valued function $\mathbf{f}_{0}$ satisfies the following inequality:

$$
\sum_{j=1}^{q} \int_{0}^{T} f_{j, 0}(t) d t \leq \frac{f_{0} \cdot \sigma}{\widehat{\zeta}} \cdot \exp \left(-\frac{q \nu T}{\sigma}\right)
$$

where $f_{j, 0}$ is the $j$ th component of $\mathbf{f}_{0}$, and $\widehat{\zeta}$ satisfies $\left\|g_{j, \epsilon_{k}}\right\|_{\infty} \leq \widehat{\zeta}$ for each $j$ and $k$. If $\alpha_{0}^{*}=0$, then $\left(\mathbf{y}_{0}^{*}, 0\right)$ is the feasible solution of problem $\left(\mathrm{AP}_{0}\right)$, and $\mathbf{y}_{0}^{*}$ and $\left(\mathbf{z}_{0}^{*}, \beta_{0}^{*}\right)$ are the optimal solutions of problems $\left(\mathrm{CLFP}_{0}\right)$ and $\left(\mathrm{DCLFP}_{0}\right)$, respectively, such that

$$
\int_{0}^{T}\left(\mathbf{f}_{0}(t)\right)^{\top} \mathbf{y}_{0}^{*}(t) d t=\beta_{0}^{*}=\frac{f_{0}+\int_{0}^{T}\left(\mathbf{f}_{0}(t)\right)^{\top} \mathbf{y}_{0}^{*}(t) d t}{h_{0}+\int_{0}^{T}(\mathbf{h}(t))^{\top} \mathbf{y}_{0}^{*}(t) d t}=\frac{f_{0}+\beta_{0}^{*}}{h_{0}+1}
$$

Proof Since $\mathbf{f}_{\epsilon_{k}}$ and $\mathbf{g}_{\epsilon_{k}}$ are assumed to be continuous on [0,T], part (i) follows from Theorem 2.2 immediately. To prove part (ii), using Proposition 4.1, there exists a subsequence $\left\{\epsilon_{k_{j}^{(\circ)}}\right\}_{j=1}^{\infty}$ of $\left\{\epsilon_{k}\right\}_{k=1}^{\infty}$ with $\epsilon_{k_{j}}^{(\circ)} \rightarrow 0+$ as $j \rightarrow \infty$ such that

$$
\lim _{j \rightarrow \infty} \beta_{\epsilon_{k_{j}}^{(\circ)}}^{*}=\beta_{0}^{*}
$$

Now, using Proposition 3.1, there exists a subsequence $\left\{\epsilon_{k_{j}}\right\}_{j=1}^{\infty}$ of $\left\{\epsilon_{k_{j}^{(\circ)}}\right\}_{j=1}^{\infty}$ with $\epsilon_{k_{j}} \rightarrow 0+$ as $j \rightarrow \infty$ such that

$$
\lim _{j \rightarrow \infty} \alpha_{\epsilon_{k_{j}}}^{*} f_{0}+\int_{0}^{T} \mathbf{f}_{\epsilon_{k_{j}}}^{\top}(t) \mathbf{y}_{\epsilon_{k_{j}}}^{*}(t) d t=\alpha_{0}^{*} f_{0}+\int_{0}^{T}\left(\mathbf{f}_{0}(t)\right)^{\top} \mathbf{y}_{0}^{*}(t) d t
$$

where the sequence $\left\{\mathbf{y}_{\epsilon_{k_{j}}}^{*}\right\}_{j=1}^{\infty}$ weakly converges to $\mathbf{y}_{0}^{*}$. From (47), we have

$$
\beta_{\epsilon_{k_{j}}}^{*}=\alpha_{\epsilon_{k_{j}}}^{*} f_{0}+\int_{0}^{T} \mathbf{f}_{\epsilon_{k_{j}}}^{\top}(t) \mathbf{y}_{\epsilon_{k_{j}}}^{*}(t) d t
$$

Therefore, using (52) and (51), we obtain the equality (48). Suppose that $\alpha_{0}^{*}>0$, by Proposition 2.1 and the equality (48), it follows that $\left(\mathbf{y}_{0}^{*}, \alpha_{0}^{*}\right)$ and $\left(\mathbf{z}_{0}^{*}, \beta_{0}^{*}\right)$ are the optimal solutions of problems $\left(\mathrm{AP}_{0}\right)$ and $\left(\mathrm{DCLFP}_{0}\right)$, respectively. On the other hand, let $\mathbf{x}_{0}^{*}=\mathbf{y}_{0}^{*} / \alpha_{0}^{*}$. Since $\mathbf{x}_{\epsilon_{k_{j}}}^{*}=\mathbf{y}_{\epsilon_{k_{j}}}^{*} / \alpha_{\epsilon_{k_{j}}}^{*}$ and the sequence $\left\{\mathbf{y}_{\epsilon_{k_{j}}}^{*}\right\}_{j=1}^{\infty}$ weakly converges to $\mathbf{y}_{0}^{*}$, it follows that

$$
\begin{aligned}
\lim _{j \rightarrow \infty} \int_{0}^{T}(\mathbf{h}(t))^{\top} \mathbf{x}_{\epsilon_{k_{j}}}^{*}(t) d t & =\left(\lim _{j \rightarrow \infty} \frac{1}{\alpha_{\epsilon_{k_{j}}}^{*}}\right) \cdot\left(\lim _{j \rightarrow \infty} \int_{0}^{T}(\mathbf{h}(t))^{\top} \mathbf{y}_{\epsilon_{k_{j}}}^{*}(t) d t\right) \\
& =\frac{1}{\alpha_{0}^{*}} \cdot \int_{0}^{T}(\mathbf{h}(t))^{\top} \mathbf{y}_{0}^{*}(t) d t=\int_{0}^{T}(\mathbf{h}(t))^{\top} \mathbf{x}_{0}^{*}(t) d t
\end{aligned}
$$

and similarly

$$
\lim _{j \rightarrow \infty} \int_{0}^{T}\left(\mathbf{f}_{0}(t)\right)^{\top} \mathbf{x}_{\epsilon_{k_{j}}}^{*}(t) d t=\int_{0}^{T}\left(\mathbf{f}_{0}(t)\right)^{\top} \mathbf{x}_{0}^{*}(t) d t
$$


Since the sequence $\left\{\mathbf{f}_{\epsilon_{k}}\right\}$ is uniformly essentially bounded on $[0, T]$, there exists a positive constant $\widehat{\tau}$ such that $\left\|f_{i, \epsilon_{k}}\right\|_{\infty} \leq \widehat{\tau}$ for each $i$ and $k$. Now we have

$$
\begin{aligned}
& \left|\int_{0}^{T}\left(\mathbf{f}_{\epsilon_{k_{j}}}(t)\right)^{\top} \mathbf{x}_{\epsilon_{k_{j}}}^{*}(t) d t-\int_{0}^{T}\left(\mathbf{f}_{0}(t)\right)^{\top} \mathbf{x}_{\epsilon_{k_{j}}}^{*}(t) d t\right| \\
& \quad=\left|\int_{0}^{T}\left[\mathbf{f}_{\epsilon_{k_{j}}}(t)-\mathbf{f}_{0}(t)\right]^{\top} \mathbf{x}_{\epsilon_{k_{j}}}^{*}(t) d t\right| \\
& \quad=\left|\int_{V_{\epsilon_{k_{j}}}}\left[\mathbf{f}_{\epsilon_{k_{j}}}(t)-\mathbf{f}_{0}(t)\right]^{\top} \mathbf{x}_{\epsilon_{k_{j}}}^{*}(t) d t\right| \quad \text { (using assumption (5)) } \\
& \quad \leq \mu\left(V_{\epsilon_{k_{j}}}\right) \cdot \sum_{i=1}^{q}\left(\widehat{\tau}+\tau_{0}\right) \cdot\left\|x_{i, \epsilon_{k_{j}}}^{*}\right\|_{\infty} \\
& \quad<q \cdot \epsilon_{k_{j}} \cdot\left(\widehat{\tau}+\tau_{0}\right) \cdot \sum_{i=1}^{q}\left\|x_{i, \epsilon_{k_{j}}}^{*}\right\|_{\infty} \quad \text { (using assumption (5)) }
\end{aligned}
$$

and

$$
\begin{aligned}
& \left|\int_{0}^{T}\left(\mathbf{f}_{\epsilon_{k_{j}}}(t)\right)^{\top} \mathbf{x}_{\epsilon_{k_{j}}}^{*}(t) d t-\int_{0}^{T}\left(\mathbf{f}_{0}(t)\right)^{\top} \mathbf{x}_{0}^{*}(t) d t\right| \\
& \leq\left|\int_{0}^{T}\left(\mathbf{f}_{\epsilon_{k_{j}}}(t)\right)^{\top} \mathbf{x}_{\epsilon_{k_{j}}}^{*}(t) d t-\int_{0}^{T}\left(\mathbf{f}_{0}(t)\right)^{\top} \mathbf{x}_{\epsilon_{k_{j}}}^{*}(t) d t\right| \\
& \quad+\left|\int_{0}^{T}\left(\mathbf{f}_{0}(t)\right)^{\top} \mathbf{x}_{\epsilon_{k_{j}}}^{*}(t) d t-\int_{0}^{T}\left(\mathbf{f}_{0}(t)\right)^{\top} \mathbf{x}_{0}^{*}(t) d t\right| \\
& \quad<q \cdot \epsilon_{k_{j}} \cdot\left(\widehat{\tau}+\tau_{0}\right) \cdot \sum_{i=1}^{q}\left\|x_{i, \epsilon_{k_{j}}}^{*}\right\|_{\infty}+\left|\int_{0}^{T}\left(\mathbf{f}_{0}(t)\right)^{\top} \mathbf{x}_{\epsilon_{k_{j}}}^{*}(t) d t-\int_{0}^{T}\left(\mathbf{f}_{0}(t)\right)^{\top} \mathbf{x}_{0}^{*}(t) d t\right| .
\end{aligned}
$$

Since $\epsilon_{k_{j}} \rightarrow 0+$ as $j \rightarrow \infty$, using (54), we obtain

$$
\lim _{j \rightarrow \infty} \int_{0}^{T} \mathbf{f}_{\epsilon_{k_{j}}}^{\top}(t) \mathbf{x}_{\epsilon_{k_{j}}}^{*}(t) d t=\int_{0}^{T}\left(\mathbf{f}_{0}(t)\right)^{\top} \mathbf{x}_{0}^{*}(t) d t .
$$

Now, using (55), (53), (47), and Proposition 4.1, we obtain the equalities (49). By the weak duality Theorem 2.1 , we conclude that $\mathbf{x}_{0}^{*}$ and $\left(\mathbf{z}_{0}^{*}, \beta_{0}^{*}\right)$ are the optimal solutions of problems $\left(\mathrm{CLFP}_{0}\right)$ and $\left(\mathrm{DCLFP}_{0}\right)$, respectively.

To prove part (iii), since $\alpha_{0}^{*}=0$, we see that $\left(\mathbf{y}_{0}^{*}, 0\right)$ is a feasible solution of problem $\left(\mathrm{AP}_{0}\right)$. Therefore we have

$$
\int_{0}^{T}(\mathbf{h}(t))^{\top} \mathbf{y}_{0}^{*}(t) d t=1
$$

and

$$
B \mathbf{y}_{0}^{*}(t) \leq \int_{0}^{t} K \mathbf{y}_{0}^{*}(s) d s \quad \text { for all } t \in[0, T],
$$

which implies

$$
B \mathbf{y}_{0}^{*}(t)-\int_{0}^{t} K \mathbf{y}_{0}^{*}(s) d s \leq 0 \leq \mathbf{g}_{0}(t) \quad \text { for all } t \in[0, T]
$$


This shows that $\mathbf{y}_{0}^{*}$ is a feasible solution of problem $\left(\mathrm{CLFP}_{0}\right)$ with the objective value

$$
\frac{f_{0}+\int_{0}^{T}\left(\mathbf{f}_{0}(t)\right)^{\top} \mathbf{y}_{0}^{*}(t) d t}{h_{0}+\int_{0}^{T}(\mathbf{h}(t))^{\top} \mathbf{y}_{0}^{*}(t) d t}=\frac{f_{0}+\int_{0}^{T}\left(\mathbf{f}_{0}(t)\right)^{\top} \mathbf{y}_{0}^{*}(t) d t}{h_{0}+1}
$$

Since $\alpha_{0}^{*}=0$, the equality (48) says that

$$
\beta_{0}^{*}=\int_{0}^{T}\left(\mathbf{f}_{0}(t)\right)^{\top} \mathbf{y}_{0}^{*}(t) d t
$$

Using (56) and weak duality Theorem 2.1, we obtain

$$
\frac{f_{0}+\beta_{0}^{*}}{h_{0}+1}=\frac{f_{0}+\int_{0}^{T}\left(\mathbf{f}_{0}(t)\right)^{\top} \mathbf{y}_{0}^{*}(t) d t}{h_{0}+1}=\frac{f_{0}+\int_{0}^{T}\left(\mathbf{f}_{0}(t)\right)^{\top} \mathbf{y}_{0}^{*}(t) d t}{h_{0}+\int_{0}^{T}(\mathbf{h}(t))^{\top} \mathbf{y}_{0}^{*}(t) d t} \leq \beta_{0}^{*} .
$$

Let $\hat{y}_{j, 0}$ be the $j$ th component of $\hat{\mathbf{y}}_{0}$. From (3) and (21), we obtain

$$
\begin{aligned}
\hat{y}_{j, 0}(t) & \leq \limsup _{i \rightarrow \infty} y_{j, \epsilon_{k_{i}}}^{*}(t) \leq \limsup _{i \rightarrow \infty} \frac{\zeta_{\epsilon_{k_{i}}}}{h_{0} \sigma} \cdot \exp \left(\frac{q \nu T}{\sigma}\right) \\
& \leq \frac{\widehat{\zeta}}{h_{0} \sigma} \cdot \exp \left(\frac{q \nu T}{\sigma}\right) \quad \text { a.e. in }[0, T] .
\end{aligned}
$$

Let $y_{j, 0}^{*}$ be the $j$ th component of $\mathbf{y}_{0}^{*}$. Using (23), we also obtain

$$
y_{j, 0}^{*}(t) \leq \frac{\widehat{\zeta}}{h_{0} \sigma} \cdot \exp \left(\frac{q v T}{\sigma}\right) \quad \text { a.e. in }[0, T]
$$

Now, using (57), (50), and (59), we have

$$
\beta_{0}^{*}=\int_{0}^{T}\left(\mathbf{f}_{0}(t)\right)^{\top} \mathbf{y}_{0}^{*}(t) d t \leq \frac{\widehat{\zeta}}{h_{0} \sigma} \cdot \exp \left(\frac{q v T}{\sigma}\right) \cdot \sum_{j=1}^{q} \int_{0}^{T} f_{j, 0}(t) d t \leq \frac{f_{0}}{h_{0}}
$$

which is equivalent to

$$
\frac{f_{0}+\beta_{0}^{*}}{h_{0}+1} \geq \beta_{0}^{*}
$$

Therefore, using (58), we obtain

$$
\frac{f_{0}+\int_{0}^{T}\left(\mathbf{f}_{0}(t)\right)^{\top} \mathbf{y}_{0}^{*}(t) d t}{h_{0}+\int_{0}^{T}(\mathbf{h}(t))^{\top} \mathbf{y}_{0}^{*}(t) d t}=\frac{f_{0}+\beta_{0}^{*}}{h_{0}+1}=\beta_{0}^{*} .
$$

By the weak duality Theorem 2.1 again, we conclude that $\mathbf{y}_{0}^{*}$ and $\left(\mathbf{z}_{0}^{*}, \beta_{0}^{*}\right)$ are the optimal solutions of problems $\left(\mathrm{CLFP}_{0}\right)$ and $\left(\mathrm{DCLFP}_{0}\right)$, respectively. This completes the proof.

\section{Strong duality theorem for the extended form}

Based on Theorem 5.1 and Lusin's theorem, we are going to extend the strong duality Theorem 2.2 by assuming the functions $\mathbf{f}$ and $\mathbf{g}$ to be measurable and bounded on $[0, T]$. 
Theorem 6.1 (Rudin [25], p.55; Lusin's theorem) Suppose that $\zeta$ is a measurable function on $X$ such that $\zeta(x)=0$ for $x \notin A$, where $A \subset X$ and $\mu(A)<\infty$. Given $\epsilon>0$, there exists a continuous function $\zeta_{\epsilon}$ on $X$ such that

$$
\mu\left(\left\{x \in X: \zeta(x) \neq \zeta_{\epsilon}(x)\right\}\right)<\epsilon
$$

Moreover, we may arrange it so that

$$
\sup _{x \in X}\left|\zeta_{\epsilon}(x)\right| \leq \sup _{x \in X}|\zeta(x)|
$$

Consider the primal and dual pair of problems (CLFP) and (DCLFP). We take $\mathbf{f}_{0}=\mathbf{f}$ and $\mathbf{g}_{0}=\mathbf{g}$. Therefore we have

$$
\left|f_{j}(t)\right| \leq \tau_{0} \quad \text { for all } t \in[0, T] \text { and for all } j=1, \ldots, q
$$

and

$$
g_{i}(t) \leq \zeta_{0} \quad \text { for all } t \in[0, T] \text { and for all } i=1, \ldots, p,
$$

where $f_{j}$ is the $j$ th component of $\mathbf{f}$ and $g_{i}$ is the $i$ th component of $\mathbf{g}$.

Lemma 6.1 Given $\epsilon>0$, there exists a vector-valued continuous function $\mathbf{f}_{\epsilon}$ on $[0, T]$ such that $V_{\epsilon}=\left\{t \in[0, T]: \mathbf{f}(t) \neq \mathbf{f}_{\epsilon}(t)\right\}$ satisfies $\mu\left(V_{\epsilon}\right)<q \cdot \epsilon$ and $\tau_{\epsilon} \leq \tau_{0}$.

Proof Since $\mathbf{f}$ is measurable on $[0, T]$ and $\mu([0, T])<\infty$, by Lusin's theorem 6.1, given $\epsilon>0$, there exists a continuous function $f_{j, \epsilon}$ such that the set

$$
V_{j, \epsilon}=\left\{t \in[0, T]: f_{j}(t) \neq f_{j, \epsilon}(t)\right\}
$$

has measure $\mu\left(V_{j, \epsilon}\right)<\epsilon$. Let $V_{\epsilon}=\bigcup_{j=1}^{q} V_{j, \epsilon}$. We define the vector-valued function

$$
\mathbf{f}_{\epsilon}(t)=\left(f_{1, \epsilon}(t), \ldots, f_{q, \epsilon}(t)\right) .
$$

Then

$$
V_{\epsilon}=\left\{t \in[0, T]: \mathbf{f}(t) \neq \mathbf{f}_{\epsilon}(t)\right\} \text { and } \quad \mu\left(V_{\epsilon}\right)<q \cdot \epsilon .
$$

According to (60), we can arrange $\mathbf{f}_{\epsilon}$ such that

$$
\sup _{t \in[0, T]}\left|f_{j, \epsilon}(t)\right| \leq \sup _{t \in[0, T]}\left|f_{j}(t)\right|
$$

for each $j=1, \ldots, q$. Since we take $\mathbf{f}_{0}=\mathbf{f}$, we have

$$
\tau_{j, \epsilon}=\max _{t \in[0, T]}\left|f_{j, \epsilon}(t)\right|=\sup _{t \in[0, T]}\left|f_{j, \epsilon}(t)\right| \leq \sup _{t \in[0, T]}\left|f_{j}(t)\right|=\sup _{t \in[0, T]}\left|f_{j, 0}(t)\right| \leq \tau_{0}
$$


for each $j=1, \ldots, q$, which says that

$$
\tau_{\epsilon}=\max \left\{\tau_{1, \epsilon}, \ldots, \tau_{q, \epsilon}\right\} \leq \tau_{0}
$$

This completes the proof.

Lemma 6.2 Given $\epsilon>0$, there exists a vector-valued continuous function $\mathbf{g}_{\epsilon}(t) \geq \mathbf{0}$ on $[0, T]$ such that $U_{\epsilon}=\left\{t \in[0, T]: \mathbf{g}(t) \neq \mathbf{g}_{\epsilon}(t)\right\}$ with $\mu\left(U_{\epsilon}\right)<p \cdot \epsilon$ and $\zeta_{\epsilon} \leq \zeta_{0}$.

Proof Since $\mathbf{g}$ is measurable on $[0, T]$ and $\mu([0, T])<\infty$, using Lusin's theorem and the similar argument from the proof of Lemma 6.1, there exists a vector-valued continuous function $\hat{\mathbf{g}}_{\epsilon}$ on $[0, T]$ such that

$$
\hat{U}_{\epsilon}=\left\{t \in[0, T]: \mathbf{g}(t) \neq \hat{\mathbf{g}}_{\epsilon}(t)\right\} \quad \text { and } \quad \mu\left(\hat{U}_{\epsilon}\right)<p \cdot \epsilon .
$$

We define $\mathbf{g}_{\epsilon}(t)=\left|\hat{\mathbf{g}}_{\epsilon}(t)\right|$. Then $\mathbf{g}_{\epsilon}(t) \geq \mathbf{0}$ for all $t \in[0, T]$ and the vector-valued function $\mathbf{g}_{\epsilon}$ is also continuous on $[0, T]$. For $t \notin \hat{U}_{\epsilon}$, we have

$$
\mathbf{g}_{\epsilon}(t)=\left|\hat{\mathbf{g}}_{\epsilon}(t)\right|=|\mathbf{g}(t)|=\mathbf{g}(t) .
$$

Equivalently, if $\mathbf{g}(t) \neq \mathbf{g}_{\epsilon}(t)$, then $t \in \hat{U}_{\epsilon}$, which says that

$$
U_{\epsilon}=\left\{t \in[0, T]: \mathbf{g}(t) \neq \mathbf{g}_{\epsilon}(t)\right\} \subseteq \hat{U}_{\epsilon} \quad \text { and } \quad \mu\left(U_{\epsilon}\right) \leq \mu\left(\hat{U}_{\epsilon}\right)<p \cdot \epsilon
$$

Finally, since we take $\mathbf{g}_{0}=\mathbf{g}$, according to (60), we can arrange $\mathbf{g}_{\epsilon}$ such that

$$
\zeta_{i, \epsilon}=\max _{t \in[0, T]}\left|g_{i, \epsilon}(t)\right|=\sup _{t \in[0, T]}\left|g_{i, \epsilon}(t)\right| \leq \sup _{t \in[0, T]}\left|g_{i}(t)\right|=\sup _{t \in[0, T]}\left|g_{i, 0}(t)\right| \leq \zeta_{0}
$$

for each $i=1, \ldots, p$, which says that $\zeta_{\epsilon} \leq \zeta_{0}$. This completes the proof.

Now, we are in a position to obtain the strong duality theorem in the extended form.

Theorem 6.2 (Strong duality theorem - extended form) Consider the primal and dual pair of problems (CLFP) and (DCLFP). Then the following statements hold true:

(i) There exist feasible solutions $\left(\mathbf{y}^{*}, \alpha^{*}\right)$ and $\left(\mathbf{z}^{*}, \beta^{*}\right)$ of problems (AP) and (DCLFP), respectively, such that

$$
\alpha^{*} f_{0}+\int_{0}^{T}(\mathbf{f}(t))^{\top} \mathbf{y}^{*}(t) d t=\beta^{*}
$$

(ii) If $\alpha^{*}>0$, then $\mathbf{x}^{*}=\mathbf{y}^{*} / \alpha^{*},\left(\mathbf{y}^{*}, \alpha^{*}\right)$, and $\left(\mathbf{z}^{*}, \beta_{0}^{*}\right)$ are the optimal solutions of problems (CLFP), (AP), and (DCLFP), respectively, such that

$$
\alpha^{*} f_{0}+\int_{0}^{T}(\mathbf{f}(t))^{\top} \mathbf{y}^{*}(t) d t=\beta^{*}=\frac{f_{0}+\int_{0}^{T}(\mathbf{f}(t))^{\top} \mathbf{x}^{*}(t) d t}{h_{0}+\int_{0}^{T}(\mathbf{h}(t))^{\top} \mathbf{x}^{*}(t) d t}
$$


(iii) We further assume that the vector-valued function $\mathbf{f}$ satisfies the following inequality:

$$
\sum_{j=1}^{q} \int_{0}^{T} f_{j}(t) d t \leq \frac{f_{0} \cdot \sigma}{\zeta_{0}} \cdot \exp \left(-\frac{q \nu T}{\sigma}\right) .
$$

If $\alpha^{*}=0$, then $\left(\mathbf{y}^{*}, 0\right)$ is the feasible solution of problem (AP), and $\mathbf{y}^{*}$ and $\left(\mathbf{z}^{*}, \beta^{*}\right)$ are the optimal solutions of problems (CLFP) and (DCLFP), respectively, such that

$$
\int_{0}^{T}(\mathbf{f}(t))^{\top} \mathbf{y}^{*}(t) d t=\beta^{*}=\frac{f_{0}+\int_{0}^{T}(\mathbf{f}(t))^{\top} \mathbf{y}^{*}(t) d t}{h_{0}+\int_{0}^{T}(\mathbf{h}(t))^{\top} \mathbf{y}^{*}(t) d t}=\frac{f_{0}+\beta^{*}}{h_{0}+1} .
$$

Proof Given a sequence $\left\{\epsilon_{k}\right\}_{k=1}^{\infty}$ in $\mathbb{R}_{+} \backslash\{0\}$ with $\epsilon_{k} \rightarrow 0+$ as $k \rightarrow \infty$, since $\mathbf{f}$ and $\mathbf{g}$ are assumed to be measurable and bounded on $[0, T]$, we can obtain the sequences of vectorvalued continuous functions $\left\{\mathbf{f}_{\epsilon_{k}}\right\}_{k=1}^{\infty}$ and $\left\{\mathbf{g}_{\epsilon_{k}}\right\}_{k=1}^{\infty}$ satisfying $\tau_{\epsilon_{k}} \leq \tau_{0}$ and $\zeta_{\epsilon_{k}} \leq \zeta_{0}$, respectively, which also says that the sequences $\left\{\mathbf{f}_{\epsilon_{k}}\right\}_{k=1}^{\infty}$ and $\left\{\mathbf{g}_{\epsilon_{k}}\right\}_{k=1}^{\infty}$ are uniformly essentially bounded on $[0, T]$. We also have $\mu\left(U_{\epsilon}\right)<p \cdot \epsilon$ and $\mu\left(V_{\epsilon}\right)<q \cdot \epsilon$ from Lemmas 6.1 and 6.2. Since $\zeta_{\epsilon} \leq \zeta_{0}$ from Lemma 6.2, it says that $\left\|g_{i, \epsilon_{k}}\right\|_{\infty} \leq \zeta_{0}$ for each $i$ and $k$. If we identify the problem (AP) with problem $\left(\mathrm{AP}_{0}\right)$, the primal problem (CLFP) with problem $\left(\mathrm{CLFP}_{0}\right)$, and the dual problem (DCLFP) with problem (DCFLP ${ }_{0}$ ) by taking $\mathbf{f}_{0}=\mathbf{f}$ and $\mathbf{g}_{0}=\mathbf{g}$, then the result follows from Theorem 5.1 by taking $\widehat{\zeta}=\zeta_{0}$. This completes the proof.

Theorem 6.3 (Strong duality theorem - extended form) Consider the primal and dual pair of problems (CLFP) and (DCLFP). Suppose that the vector-valued function $\mathbf{f}$ satisfies the following inequality:

$$
\sum_{j=1}^{q} \int_{0}^{T} f_{j}(t) d t \leq \frac{f_{0} \cdot \sigma}{\zeta_{0}} \cdot \exp \left(-\frac{q \nu T}{\sigma}\right)
$$

Then the primal and dual pair of problems (CLFP) and (DCLFP) have no duality gap.

Proof The result follows from parts (ii) and (iii) of Theorem 6.2 immediately.

Since the vector-valued function $\mathbf{f}$ is bounded by $\tau_{0}$, i.e., $\left|f_{j}(t)\right| \leq \tau_{0}$ for all $t \in[0, T]$ and for all $j=1, \ldots, q$, we also have the following interesting result.

Theorem 6.4 (Strong duality theorem - extended form) Consider the primal and dual pair of problems (CLFP) and (DCLFP). Suppose that the bound $\tau_{0}$ satisfies

$$
\tau_{0} \leq \frac{f_{0} \cdot \sigma}{q \cdot T \cdot \zeta_{0}} \cdot \exp \left(-\frac{q v T}{\sigma}\right) .
$$

Then the primal and dual pair of problems (CLFP) and (DCLFP) have no duality gap.

Proof Since

$$
\sum_{j=1}^{q} \int_{0}^{T} f_{j}(t) d t \leq \sum_{j=1}^{q} \int_{0}^{T}\left|f_{j}(t)\right| d t \leq q \cdot T \cdot \tau_{0},
$$


the inequality (61) is satisfied automatically by using (62). The result follows from Theorem 6.3 immediately.

\section{Competing interests}

The author declares that he has no competing interests.

Received: 15 May 2015 Accepted: 30 July 2015 Published online: 19 August 2015

\section{References}

1. Tyndall, WF: A duality theorem for a class of continuous linear programming problems. SIAM J. Appl. Math. 15 644-666 (1965)

2. Tyndall, WF: An extended duality theorem for continuous linear programming problems. SIAM J. Appl. Math. 15 , 1294-1298 (1967)

3. Bellman, RE: Dynamic Programming. Princeton University Press, Princeton (1957)

4. Levinson, N: A class of continuous linear programming problems. J. Math. Anal. Appl. 16, 73-83 (1966)

5. Meidan, R, Perold, AF: Optimality conditions and strong duality in abstract and continuous-time linear programming. J. Optim. Theory Appl. 40, 61-77 (1983)

6. Papageorgiou, NS: A class of infinite dimensional linear programming problems. J. Math. Anal. Appl. 87, 228-245 (1982)

7. Schechter, M: Duality in continuous linear programming. J. Math. Anal. Appl. 37, 130-141 (1972)

8. Anderson, EJ, Nash, P, Perold, AF: Some properties of a class of continuous linear programs. SIAM J. Control Optim. 21, 758-765 (1983)

9. Anderson, EJ, Philpott, AB: On the solutions of a class of continuous linear programs. SIAM J. Control Optim. 32, 1289-1296 (1994)

10. Anderson, EJ, Pullan, MC: Purification for separated continuous linear programs. Math. Methods Oper. Res. 43, 9-33 (1996)

11. Fleischer, L, Sethuraman, J: Efficient algorithms for separated continuous linear programs: the multicommodity flow problem with holding costs and extensions. Math. Oper. Res. 30, 916-938 (2005)

12. Pullan, MC: An algorithm for a class of continuous linear programs. SIAM J. Control Optim. 31, 1558-1577 (1993)

13. Pullan, MC: Forms of optimal solutions for separated continuous linear programs. SIAM J. Control Optim. 33, 1952-1977 (1995)

14. Pullan, MC: A duality theory for separated continuous linear programs. SIAM J. Control Optim. 34, $931-965$ (1996)

15. Pullan, MC: Convergence of a general class of algorithms for separated continuous linear programs. SIAM J. Optim. 10, $722-731(2000)$

16. Pullan, MC: An extended algorithm for separated continuous linear programs. Math. Program., Ser. A 93, 415-451 (2002)

17. Weiss, G: A simplex based algorithm to solve separated continuous linear programs. Math. Program., Ser. A 115 151-198 (2008)

18. Zalmai, GJ: Duality for a class of continuous-time homogeneous fractional programming problems. Z. Oper.-Res. 30, 43-48 (1986)

19. Zalmai, GJ: Duality for a class of continuous-time fractional programming problems. Util. Math. 31, $209-218$ (1987)

20. Zalmai, GJ: Optimality conditions and duality for a class of continuous-time generalized fractional programming problems. J. Math. Anal. Appl. 153, 365-371 (1990)

21. Zalmai, GJ: Optimality conditions and duality models for a class of nonsmooth constrained fractional optimal control problems. J. Math. Anal. Appl. 210, 114-149 (1997)

22. Wen, $\mathrm{C}-\mathrm{F}, \mathrm{Wu}, \mathrm{H}-\mathrm{C}$ : Approximate solutions and duality theorems for continuous-time linear fractional programming problems. Numer. Funct. Anal. Optim. 33, 80-129 (2012)

23. Charnes, A, Cooper, WW: Programming with linear fractional functions. Nav. Res. Logist. Q. 9, 181-186 (1962)

24. Riesz, F, Sz.-Nagy, B: Functional Analysis. Ungar, New York (1955)

25. Rudin, W: Real and Complex Analysis, 3rd edn. McGraw-Hill, New York (1986)

\section{Submit your manuscript to a SpringerOpen ${ }^{\circ}$ journal and benefit from:}

- Convenient online submission

Rigorous peer review

- Immediate publication on acceptance

- Open access: articles freely available online

- High visibility within the field

- Retaining the copyright to your article

Submit your next manuscript at $>$ springeropen.com 